\title{
Modified Maximum Likelihood Estimation from Censored Samples in Burr Type X Distribution
}

\author{
R.R.L. Kantam \\ Department of Statistics, Acharya Nagarjuna University \\ Nagarjunanagar-522510, Guntur-Andhra Pradesh, India \\ kantam.rrl@gmail.com
}

\author{
M.S. Ravikumar \\ Department of Community Medicine \\ Konaseema Institute of Medical Sciences \& Research Foundation/ \\ General Hospital, Amalapuram-533201, Andhra Pradesh, India \\ msrk.raama@gmail.com
}

\begin{abstract}
The two parameter Burr type $\mathrm{X}$ distribution is considered and its scale parameter is estimated from a censored sample using the classical maximum likelihood method. The estimating equations are modified to get simpler and efficient estimators. Two methods of modification are suggested. The small sample efficiencies are presented.
\end{abstract}

Keywords: MLE, Censored Samples, Order Statistics, Asymptotic Variance.

\section{Introduction}

In reliability studies exponential distribution is the central model for the study of any phenomenon through a probabilistic approach. Extension of this model into two different directions yields two popular models called the Weibull distribution and the gamma distribution. Between these two, the Weibull distribution is more frequently applied model in any practical situation concerning reliability studies. Both Weibull and gamma distributions involve a shape parameter, in the sense that the shape of the frequency curve of these models changes according as the change in the values of the shape parameters. In particular, for the Weibull distribution its shape parameter classifies it into an Increasing Failure Rate (IFR) model or a Decreasing Failure Rate (DFR) model or Constant Failure Rate (CFR) model (the well known exponential model) as its special cases. The natural phenomenon in reliability is "The aging concept" - indicated by increasing instantaneous failure probability with age of the product. A specific case of Weibull distribution exhibiting aging effect with an integer valued shape parameter is known as "The Rayleigh distribution". Its cumulative distribution function (CDF), probability density function (PDF) and hazard function are given by

$$
\begin{aligned}
& F(x)=1-e^{-x^{2}} \\
& f(x)=2 x e^{-x^{2}} \\
& h(x)=2 x
\end{aligned}
$$

If $\mathrm{F}(\mathrm{x})$ is the cumulative distribution function of a positive valued random variable, then $[F(x)]^{k}, \mathrm{k}>0$ also satisfies all the requirements for the cumulative distribution function of another positive valued random variable. It can be interpreted as the failure probability of 
a parallel system of $\mathrm{k}$ - components whose life times are independently and identically distributed random variables, each with a common CDF $-\mathrm{F}(x)$ if $k$ is an integer. Exploring this concept to non integer values of $k$ also, many researchers in the recent past made extensive studies on models of the type $[F(x)]^{k}$ generated by a basic well known model $\mathrm{F}(x)$. Such new models are named as exponentiated models by some authors and generalised models by some authors. For instance if the basic $\mathrm{F}(x)$ is exponential, $[F(x)]^{k}$ is named as generalised exponential (Gupta \& Kundu- 1999), if $F(x)$ is Weibull, $[F(x)]^{k}$ is named as exponentiated Weibull (Mudholkar and Srivastava - 1993). Banking on this notion, generalised Rayleigh distribution was studied by Raqab and Kundu (2006), as a process of revisit to Burr type X distribution. Its cumulative distribution function $(\mathrm{CDF})$, probability density function (PDF) and hazard function are given by

$$
\begin{aligned}
& F(x ; k)=\left(1-e^{-x^{2}}\right)^{k} ; x>0, k>0 \\
& f(x ; k)=2 k x e^{-x^{2}}\left(1-e^{-x^{2}}\right)^{k-1} ; x>0, k>0 \\
& h(x ; k)=\frac{2 k x e^{-x^{2}}\left(1-e^{-x^{2}}\right)^{(k-1)}}{1-\left(1-e^{-x^{2}}\right)^{k}}
\end{aligned}
$$

Burr (1942) has suggested a number of forms of cumulative distribution functions that might be useful in modeling various practical situations. In all, he suggested twelve models as listed below.

(II) $\quad F(x)=\left(e^{-x}+1\right)^{-k}$,

(III) $\quad F(x)=\left(x^{-c}+1\right)^{-k} ; 0<x<\infty$,

(IV) $\quad F(x)=\left[\left(\frac{c-x}{x}\right)^{1 / c}+1\right]^{-k} ; 0<x<c$,

(V) $\quad F(x)=\left(c e^{-\tan x}+1\right)^{-k} ;-\frac{\pi}{2}<x<\frac{\pi}{2}$,

(VI) $\quad F(x)=\left(c e^{-k \sinh x}+1\right)^{-k}$,

(VII) $\quad F(x)=2^{-k}(1+\tanh x)^{k}$,

(VIII) $\quad F(x)=\left(\frac{2}{\pi} \arctan e^{x}\right)^{k}$,

(IX) $\quad F(x)=1-\frac{2}{c\left[\left(1+e^{x}\right)^{k}-1\right]+2}$,

(X) $\quad F(x)=\left(1-e^{-x^{2}}\right)^{k} ; 0<x<\infty$,

(XI) $\quad F(x)=\left(x-\frac{1}{2 \pi} \sin 2 \pi x\right)^{k} ; 0<x<1$,

(XII) $\quad F(x)=1-\left(1+x^{c}\right)^{-k} ; 0<x<\infty$.

Thus generalised Rayleigh distribution and Burr type $\mathrm{X}$ distributions are one and the same. 
In the above models $k$ and $c$ are the positive parameters involved in the respective models. Among these twelve forms, the type X and type XII models are most frequently applied by many researchers. Our focus is Burr Type X model, whose expressions are given in equations (1.4), (1.5), and (1.6). If a scale parameter say $\lambda$ is introduced, the cumulative distribution function, probability density function and hazard function are given by

$$
\begin{aligned}
& F(x ; k, \lambda)=\left(1-e^{-(\lambda x)^{2}}\right)^{k} ; x>0, k>0, \lambda>0, \\
& f(x ; k, \lambda)=2 k \lambda^{2} x e^{-(\lambda x)^{2}}\left(1-e^{-(\lambda x)^{2}}\right)^{(k-1)} ; x>0, k>0, \lambda>0, \\
& h(x ; k, \lambda)=\frac{2 k \lambda^{2} x e^{-(\lambda x)^{2}\left(1-e^{\left.-(\lambda x)^{2}\right)^{(k-1)}}\right.}}{1-\left(1-e^{-(\lambda x)^{2}}\right)^{k}} .
\end{aligned}
$$

Expressions in (1.4), (1.5) and (1.6) are called standard Burr type X model, those in equations (1.8), (1.9) and (1.10) are called scaled Burr type X or Two parameter Burr type X model.

In this paper estimation of $\lambda$ from type II censored sample where $k$ is known is studied. Kantam and Ravikumar (2015) obtained modified maximum likelihood estimation (MMLE) of $\lambda$ from complete samples. For a ready reference estimation from complete samples is presented in Section -2 . We discuss estimation from censored samples in Section - 3. This section includes the asymptotic as well as small sample behavior of the estimates. As these processes involve a lot of numerical computations, we present our results in the forms various numerical tables towards the end of respective sections of this paper with the respective labels of identification.

\section{Estimation from Complete Samples}

The probability density function of the two parameter Burr type $\mathrm{X}$ distribution is given by $f(x ; k, \lambda)=2 k \lambda^{2} x e^{-(\lambda x)^{2}}\left(1-e^{-(\lambda x)^{2}}\right)^{(k-1)} ; x>0, k>0, \lambda>0$

Let $x_{1}<x_{2}<x_{3}<\ldots<x_{n}$ be a complete ordered sample of size $n$ drawn from the above distribution (Though for complete samples maximum likelihood estimation does not require ordering of the sample, in order to facilitate presenting the methods to be introduced for this Section, which depend on ordered samples we consider ordered complete samples in the beginning itself, for uniformity in the notation.). The $\log$ likelihood equations to get the maximum likelihood estimates of $\lambda$ and $k$ are given by (after simplification).

$\frac{\partial \log L}{\partial \lambda}=0 \Rightarrow \frac{2 n}{\lambda}-2 \lambda \sum_{i=1}^{n} x_{i}^{2}+2 \lambda(k-1) \sum_{i=1}^{n} \frac{x_{i}^{2} e^{-\left(\lambda x_{i}\right)^{2}}}{1-e^{-\left(\lambda x_{i}\right)^{2}}}=0$
$\frac{\partial \log L}{\partial k}=0 \Rightarrow \frac{n}{k}+\sum_{i=1}^{n} \ln \left(1-e^{-\left(\lambda x_{i}\right)^{2}}\right)=0$

These equations show that maximum likelihood estimator of $\lambda$ is an iterative solution involving $k$ and maximum likelihood estimator of $k$ is a closed form expression involving $\lambda$ given as

$\hat{k}=\frac{-n}{\sum_{i=1}^{n} \ln \left(1-e^{-\left(\lambda x_{i}\right)^{2}}\right)}$ 
Since $\lambda$ is a scale parameter, in view of the scale invariant nature of the Burr type $X$ distribution, maximum likelihood estimation of $k$ can be taken as the following expression in a standard model.

$\hat{k}=\frac{-n}{\sum_{i=1}^{n} \ln \left(1-e^{-z_{i}^{2}}\right)}$

where $\lambda x_{i}=z_{i}$.

The elements of the information matrix and hence those of the asymptotic dispersion matrix require the following second order partial derivatives.

$\frac{\partial^{2} \log f(x ; \lambda, k)}{\partial \lambda^{2}}=\frac{-2}{\lambda^{2}}-2 x^{2}+\frac{2 x^{2}(k-1) e^{-(\lambda x)^{2}}\left(1-2 \lambda^{2} x^{2}-e^{-(\lambda x)^{2}}\right)}{\left[1-e^{-(\lambda x)^{2}}\right]^{2}}$

$\frac{\partial^{2} \log f(x ; \lambda, k)}{\partial k^{2}}=\frac{-1}{k^{2}}$

$\frac{\partial^{2} \log f(x ; \lambda, k)}{\partial \lambda \partial k}=\frac{2 x^{2} \lambda e^{-(\lambda x)^{2}}}{1-e^{-(\lambda x)^{2}}}$

The information matrix is given by

$\left[\begin{array}{ll}-n E\left[\frac{\partial^{2} \log f(x ; \lambda, k)}{\partial \lambda^{2}}\right] & -n E\left[\frac{\partial^{2} \log f(x ; \lambda, k)}{\partial \lambda \partial k}\right] \\ -n E\left[\frac{\partial^{2} \log f(x ; \lambda, k)}{\partial \lambda \partial k}\right] & -n E\left[\frac{\partial^{2} \log f(x ; \lambda, k)}{\partial k^{2}}\right]\end{array}\right]$

As the integrals involved in these mathematical expectations are not analytically tractable we have evaluated them using 11- point Gauss-Laguerre quadrature formula (Rao et al., 1966) for selected values of $k$ in a standard density $(\lambda=1)$ and are given in Table - 2.1. The elements of the corresponding asymptotic dispersion matrix for selected values of $k$ are given in Table -2.2 .

Table 2.1: Elements to get Information Matrix at $k=2$ and 3

\begin{tabular}{|c|l|l|l|l|l|l|}
\hline $\boldsymbol{k}$ & \multicolumn{4}{|l|}{$\mathbf{2}$} & $\mathbf{3}$ & \\
\hline \multirow{2}{*}{ Matrix } & $\mathbf{I}_{\mathbf{1 1}}$ & $\mathbf{I}_{\mathbf{1 2}}$ & $\mathbf{I}_{\mathbf{2 2}}$ & $\mathbf{I}_{\mathbf{1 1}}$ & $\mathbf{I}_{\mathbf{1 2}}$ & $\mathbf{I}_{\mathbf{2 2}}$ \\
\cline { 2 - 7 } & 8.84668 & -0.989225 & 0.25 & 10.9596 & -0.68296 & 0.1111 \\
\hline
\end{tabular}

Table 2.2: Elements of Asymptotic Dispersion Matrix of MLEs of $\lambda \& k$

\begin{tabular}{|l|c|c|c|c|c|c|}
\hline \multirow{2}{*}{$\boldsymbol{n}$} & $\boldsymbol{k}$ & \multicolumn{6}{l|}{$\boldsymbol{2}$} & \multicolumn{3}{l|}{$\mathbf{3}$} \\
\cline { 2 - 7 } & $\boldsymbol{\sigma}_{\mathbf{1 1}}$ & $\boldsymbol{\sigma}_{\mathbf{1 2}}$ & $\boldsymbol{\sigma}_{\mathbf{2 2}}$ & $\boldsymbol{\sigma}_{\mathbf{1 1}}$ & $\boldsymbol{\sigma}_{\mathbf{1 2}}$ & $\boldsymbol{\sigma}_{\mathbf{2 2}}$ \\
\hline 5 & 0.040548 & 0.160445 & 1.434864 & 0.029579 & 0.181811 & 2.917553 \\
\hline 10 & 0.020274 & 0.080222 & 0.717432 & 0.014789 & 0.090905 & 1.458777 \\
\hline 15 & 0.013516 & 0.053482 & 0.478288 & 0.00986 & 0.060604 & 0.972518 \\
\hline 20 & 0.010137 & 0.040111 & 0.358716 & 0.007395 & 0.045453 & 0.729388 \\
\hline 25 & 0.00811 & 0.032089 & 0.286973 & 0.005916 & 0.036362 & 0.583511 \\
\hline
\end{tabular}


When the log likelihood equations do not admit analytical expressions as MLEs of the parameters of a density function from complete or censored sample, replacement of certain portions of $\log$ likelihood equations by suitable admissible approximations sometimes would lead to simpler and efficient estimates of the parameters. Such estimates in literature are named as approximate or modified MLEs. Tiku (1967); Mehrotra and Nanda (1974); Pearson and Rootzen (1977); Tiku and Suresh (1992); Rosaiah et al. (1993a); Rosaiah et al. (1993b); Kantam and Srinivasa Rao (1993); Kantam and Srinivasa Rao (2002); Kantam and Sriram (2003); Kantam et al. (2013) and the references therein are some of the works in this direction. We adopt this concept of MML estimation for Burr type $\mathrm{X}$ distribution by considering its reparameterised version as given in Raqab and Kundu (2006).

We see that the maximum likelihood estimator of the shape parameter $k$ is a closed form expression whereas, the maximum likelihood estimator of $\lambda$ is an iterative solution of the equation (2.2) involving $k$. In order to overcome the iterative nature of the solution we proceed as follows. Equation (2.2) can be rewritten as

$2 n-2 \sum_{i=1}^{n} z_{i}^{2}+2(k-1) \sum_{i=1}^{n} \frac{z_{i}^{2} e^{-z_{i}^{2}}}{1-e^{-z_{i}^{2}}}=0$,

where $z_{i}=\lambda x_{i}$.

Consider the expression $g\left(z_{i}\right)=\frac{z_{i}^{2} e^{-z_{i}^{2}}}{1-e^{-z_{i}^{2}}}$

of equation (2.9).

We approximate this expression by a linear one in $z_{i}$ in a small neighborhood of the $i^{\text {th }}$ quantile of the population say

$g\left(z_{i}\right) \cong \alpha_{i}+\beta_{i} z_{i}$

With this approximation, equation (2.9) becomes a quadratic equation in $\lambda$ given by

$A \lambda^{2}+B \lambda+C=0$.

where, $A=\sum_{i=1}^{n} z_{i}^{2}, B=-(k-1) \sum_{i=1}^{n} \beta_{i} z_{i}, \quad C=-n-(k-1) \sum_{i=1}^{n} \alpha_{i}$.

Positive root of this equation is an estimate of $\lambda$ called the modified maximum likelihood estimate (MMLE) of $\lambda$. It can be seen that A,B,C depend on the ordered observations $x_{1}, x_{2}, \ldots, x_{n}$, the shape parameter $k$ and the slope, intercept of the linear approximation (2.11). In order to find $\alpha_{i}, \beta_{i}$, we suggest two methods.

\section{Method-I:}

Let $p_{i}=\frac{i}{n+1}, i=1,2, \ldots n$.

Let $z_{i}^{*}, z_{i}^{* *}$ be the solutions of the following equations

$F\left(z_{i}^{*}\right)=p_{i}^{*}, F\left(z_{i}^{* *}\right)=p_{i}^{* *}$,

where $p_{i}^{*}=p_{i}-\sqrt{\frac{p_{i} q_{i}}{n}}, p_{i}^{* *}=p_{i}+\sqrt{\frac{p_{i} q_{i}}{n}}, \mathrm{~F}($.$) is the cdf of standard Burr type \mathrm{X}$ distribution, and $q_{i}=1-p_{i}$. 
The expressions for $z_{i}^{*}, z_{i}^{* *}$ are

$$
\begin{aligned}
& z_{i}^{*}=\sqrt{-\operatorname{Ln}\left[1-\left(p_{i}-\sqrt{\frac{p_{i} q_{i}}{n}}\right)^{k}\right]}, \\
& z_{i}^{* *}=\sqrt{-\operatorname{Ln}\left[1-\left(p_{i}+\sqrt{\frac{p_{i} q_{i}}{n}}\right)^{k}\right]}
\end{aligned}
$$

The slope $\beta_{i}$ and intercept $\alpha_{i}$ of the linear approximation in the equation (2.11) are given by

$\beta_{i}=\frac{g\left(Z_{i}^{* *}\right)-g\left(Z_{i}^{*}\right)}{Z_{i}^{* *}-Z_{i}^{*}}$

$\alpha_{i}=g\left(z_{i}^{*}\right)-\beta_{i} z_{i}^{*}$.

where $\beta_{i}$ is given by (2.15). The values of $\alpha_{i}$ and $\beta_{i}$ in this method for $n=5(5) 25$ for $k=0.25,0.50,1.50,2,2.5,3$ are given in Table 2.3 in Appendix-I.

\section{Method-II:}

Considering Taylor's expansion of $g(z i)$ upto its first derivative w.r.t $\mathrm{z}_{i}$ in a neighborhood of population quantile corresponding to $p_{i}$, we get

$\beta_{i}=g^{\prime}\left(z_{i}\right)$,

$\alpha_{i}=g\left(z_{i}\right)-\beta_{i} z_{i}$,

where $z_{i}$ is the quantile of Burr type $\mathrm{X}$ distribution, given as the solution of the equation $F\left(z_{i}\right)=p_{i}$.

i.e., $z_{i}=\sqrt{-\operatorname{Ln}\left[1-\left(p_{i}-\sqrt{\frac{p_{i} q_{i}}{n}}\right)^{k}\right]}$

It can be seen from (2.10) that

$g^{\prime}\left(z_{i}\right)=\frac{2 z_{i} e^{-z_{i}^{2}}\left(1-z_{i}-e^{-z_{i}^{2}}+z_{i} e^{-z_{i}^{2}}-z_{i}^{2} e^{-z_{i}^{2}}\right)}{\left(1-e^{-z_{i}^{2}}\right)^{2}}$

Substituting (2.19) in (2.20) we get

$\beta_{i}=g^{\prime}\left(z_{i}\right)=\frac{2 z_{i} e^{-z_{i}^{2}}\left(1-z_{i}-e^{-z_{i}^{2}}+z_{i} e^{-z_{i}^{2}}-z_{i}^{2} e^{-z_{i}^{2}}\right)}{\left(1-e^{-z_{i}^{2}}\right)^{2}}$

Using $\beta_{i}$ in (2.18) we get $\alpha_{i}$.

The values of $\alpha_{i}$ and $\beta_{i}$ in this method for $n=5(5) 25$ for $k=0.25,0.50,1.5,2,2.5,3$ are given in Table 2.4 in Appendix-II.

In the above two modified methods, the basic principle is that certain expressions in the log likelihood equation are linearised in a neighborhood of the population quantile which 
depends on the size of the sample also. The larger the size, the narrower is the neighborhood and hence the closer is the approximation. That is, the exactness of the approximation becomes finer and finer for large values of ' $n$ '. Hence the approximate log likelihood equation and the exact log likelihood equation tend to each other as $n \rightarrow \infty$. Hence the exact and modified MLEs are asymptotically identical (Tiku et al. 1986). The same may not be true in small samples and these are to be assessed with the help of small sample characteristics of the MMLEs. Because of non-tractability of analytical sampling variances, we compared the modified ML method with exact ML method through Monte-Carlo simulation.

10,000 random samples of size $n=5$ (5) 25 each are generated from Burr type $X$ distribution with $k=0.25,0.50,1.5,2,2.5,3$ in succession. For each sample at a given $k$ the $\alpha_{i}$ and $\beta_{i}$ of Method-I (Method-II) as given in Table 2.3 (2.4) are used in equation 2.12 to get the modified MLE of ' $\lambda$ ' by Method-I (Method-II). The empirical variances of MMLEs by Method-I and Method-II are respectively given in Table 2.5 in Appendix III.

\section{Estimation from Censored Samples}

In life testing experiments censoring a given sample sometimes becomes necessary to save time and cost of experimentation. One common scheme of censoring is a failure censored sample or a type II right censored sample, wherein pre-planned $n$ items are put to life testing and the experiment will be terminated as soon as a prefixed observations say ' $\mathrm{r}$ ' are noted down $(\mathrm{r}<\mathrm{n})$. In such situations, we are left with ' $\mathrm{r}$ ' actual observations say $x_{1}<x_{2}<\cdots<x_{r}$ and the life times of the remaining $(n-r)$ items are more than $x_{r}$. Such a sample is called type - II right censored sample.

In this Section, we assume that we have a type - II right censored sample modeled by Burr type $\mathrm{X}$ distribution with shape parameter ' $k$ ' and scale parameter ' $\lambda$ '. Maximum likelihood estimation of scale parameter for a known shape parameter will be discussed below.

Let $x_{1}<x_{2}<\cdots<x_{r}$ be a type - II right censored sample from a Burr type $\mathrm{X}$ distribution in a planned random sample of sized ' $n$ '. The likelihood function of such a censored sample is given by $\alpha$ is

$$
\prod_{i=1}^{r} f\left(x_{i} ; \lambda, k\right) \cdot\left[1-F\left(x_{r} ; \lambda, k\right)\right]^{n-r}
$$

where $f(\cdot), F(\cdot)$ respectively denote the probability density function and cumulative distribution function of Burr type $\mathrm{X}$ distribution. Substituting the respective expressions for $f, F$, taking natural logarithms, differentiating with respect to ' $\lambda$ ' and on simplification we get the estimating equation for the parameter ' $\lambda$ ' as

$r-\sum_{i=1}^{r} z_{i}^{2}+(k-1) \sum_{i=1}^{r} \frac{z_{i}^{2} e^{-z_{i}^{2}}}{\left(1-e^{-z_{i}^{2}}\right)}+\frac{k^{2}(n-r) z_{r}^{2} e^{-z_{r}^{2}}\left(1-e^{-z_{r}^{2}}\right)^{(k-1)}}{1-\left(1-e^{-z_{r}^{2}}\right)^{k}}=0$,

where $z_{i}=\lambda x_{i}$ 
It can be seen that for known value of $k$, the MLE of $\lambda$ from censored sample is an iterative solution of equation (3.2). However, an analytical solution can be obtained by admissible modifications to some terms of equation (3.2) on lines of the procedures described in Section - 2. Consider the following two expressions of equation (3.2).

$g\left(z_{i}\right)=\frac{z_{i}^{2} e^{-z_{i}^{2}}}{1-e^{-z_{i}^{2}}}$

$h\left(z_{r}\right)=\frac{z_{r}^{2} e^{-z_{r}^{2}}\left(1-e^{-z_{r}^{2}}\right)^{(k-1)}}{1-\left(1-e^{-z_{r}^{2}}\right)^{k}}$

We suggest to approximate $g\left(z_{i}\right)$ of (4.3), $h\left(z_{r}\right)$ of (3.4) by the following linear expressions in small neighborhoods of $i^{\text {th }}$ population quantile, $r^{\text {th }}$ population quantile respectively.

i.e., $g\left(z_{i}\right) \cong \alpha_{i}+\beta_{i} z_{i}$

$h\left(z_{r}\right) \cong Y_{r}+\delta_{r} z_{r}$

Substituting these approximations in equation (3.2) and on simplification we get the following quadratic equation in ' $\lambda$ '.

$A \lambda^{2}+B \lambda+C=0$

where $A=-\sum_{i=1}^{r} x_{i}^{2}$

$$
\begin{gathered}
B=(k-1) \sum_{i=1}^{r} x_{i} \beta_{i}+k^{2}(n-r) \delta_{r} x_{r} \\
C=r+(k-1) \sum_{i=1}^{r} \alpha_{i}+k^{2}(n-r) Y_{r}
\end{gathered}
$$

Positive root of the above quadratic equation in (3.7) is taken as an estimate of $\lambda$ called MMLE of $\lambda$ from censored samples. We attempt to support our linear approximations for $h\left(z_{r}\right)$ in the following way.

Let $p=\frac{r+1}{n+1} ; \quad q=1-p$

$p^{*}=p-\sqrt{\frac{p q}{n}} ; \quad p^{* *}=p+\sqrt{\frac{p q}{n}}$

$z^{*}$ be the solution of $F(x)=p^{*}$

$z^{* *}$ be the solution of $F(x)=p^{* *}$ 
The interval $\left(z^{*}, z^{* *}\right)$ is evenly divided by 10 cut off points say $z^{*}=z_{1}<z_{2}<\ldots<z_{10}=z^{* *}$. The Karl-Pearson's product moment correlation coefficient between $\left(z_{i}, h\left(z_{i}\right)\right), i=1,2$, $3 \ldots 10$ is calculated for $n=5(5) 25$ with all possible values of ' $r$ '. These are given in Table - 3.1 in Appendix - III, which show that there is a high linear relation between $z$ and $h(z)$ indicating an acceptable linear approximation for $h(z)$ in the neighborhood $z_{r}$ as expressed in the equation

$h\left(z_{r}\right) \cong Y_{r}+\delta_{r} z_{r}$

It can be seen that the constants A,B,C of the above quadratic equation in (3.7) depend on the values of the uncensored observations, size of the planned sample, number of available observations, the known shape parameter $k$, the slopes and intercepts of the linear approximations for $g\left(z_{i}\right)$ of (3.5) and $h\left(z_{r}\right)$ of (3.6). We suggest as in Section - 2, two methods of finding these slopes and intercepts. It may also be noted that the slope $\left(\beta_{i}\right)$, the intercept $\left(\alpha_{i}\right)$ in the linear approximation for $g\left(z_{i}\right)$ remain unaltered from those of Section - 2. The slope $\left(\delta_{r}\right)$ and the intercept $\left(\Upsilon_{r}\right)$ of the linear approximation of $h\left(z_{r}\right)$ will now be evaluated by methods - I and II of Section -2 .

\section{Method - I:}

Let $p_{r}=\frac{r}{n+1}, q_{r}=1-p_{r}$.

$p_{r}^{\prime}=p_{r}-\sqrt{\frac{p_{r} q_{r}}{n}}, \quad p_{r}^{\prime \prime}=p_{r}+\sqrt{\frac{p_{r} q_{r}}{n}}$

Let $\xi_{r}^{\prime}, \xi_{r}^{\prime \prime}$ be the respective solutions of $F\left(x_{r}\right)=p_{r}^{\prime}, F\left(x_{r}\right)=p_{r}^{\prime \prime}$, where $\mathrm{F}($.$) is the CDF of Burr type \mathrm{X}$ distribution.

i.e., $\xi_{r}^{\prime}=\sqrt{-\operatorname{Ln}\left(1-\left(p_{r}^{\prime}\right)^{1 / k}\right)}$

$\xi_{r}^{\prime \prime}=\sqrt{-\operatorname{Ln}\left(1-\left(p_{r}^{\prime \prime}\right)^{1 / k}\right)}$

The slope $\delta_{r}=\frac{h\left(\xi_{r}^{\prime \prime}\right)-h\left(\xi_{r}^{\prime}\right)}{\xi_{r}^{\prime \prime}-\xi_{r}^{\prime}}$

and intercept $\Upsilon_{r}=h\left(\xi_{r}^{\prime \prime}\right)-\delta_{r} \xi_{r}^{\prime \prime}$

The values of $Y_{r}, \delta_{r}$ for $n=5(5) 25$, for all possible values of $r$ within each ' $n$ ' are tabulated in Tables 3.2 to 3.7 in Appendix-IV. 


\section{Method - II:}

Expanding $h\left(z_{r}\right)$ in Taylor series up to the first derivative in the neighborhood of the $r^{\text {th }}$ quantile of the population and after some simplification we get

$\delta_{r}=h^{\prime}\left(\xi_{r}\right)$

From equation (3.4) we get that

$$
\begin{aligned}
& h^{\prime}\left(z_{r}\right)= \\
& \frac{2 z e^{-z^{2}}\left(1-e^{-z^{2}}\right)^{k-1}\left[\left(1-\left(1-e^{-z^{2}}\right)^{k}\right)\left\{(k-1) z^{2} e^{-z^{2}}\left(1-e^{-z^{2}}\right)^{-1}+\left(1-z^{2}\right)\right\}+\left\{k z^{2} e^{-z^{2}}\left(1-e^{-z^{2}}\right)^{k-1}\right\}\right]}{\left[1-\left(1-e^{-z^{2}}\right)^{k}\right]^{2}}
\end{aligned}
$$

Replacing $\mathrm{z}$ with $\xi_{r}$ in equation (3.17) we get

$\delta_{r}=h^{\prime}\left(\xi_{r}\right)=$

$\frac{2 \xi_{r} e^{-\xi_{r}^{2}}\left(1-e^{-\xi_{r}^{2}}\right)^{k-1}\left[\left(1-\left(1-e^{-\xi_{r}^{2}}\right)^{k}\right)\left\{(k-1) \xi_{r}^{2} e^{-\xi_{r}^{2}}\left(1-e^{-\xi_{r}^{2}}\right)^{-1}+\left(1-\xi_{r}^{2}\right)\right\}+\left\{k \xi_{r}^{2} e^{\left.\left.-\xi_{r}^{2}\left(1-e^{-\xi_{r}^{2}}\right)^{k-1}\right\}\right]}\right.\right.}{\left[1-\left(1-e^{-\xi_{r}^{2}}\right)^{k}\right]^{2}}(3$

Let $F(x)=p=\frac{r}{n+1}$.

Then $\xi_{r}=z=\sqrt{-\operatorname{Ln}\left[1-\left(\frac{r}{n+1}\right)^{1 / k}\right]}$

$\Upsilon_{r}=h\left(\xi_{r}\right)-\delta_{r} \xi_{r}$

The values of $Y_{r}, \delta_{r}$ for $n=5(5) 25$ and all possible values of $\boldsymbol{r}$ are given in Tables 3.8 to 3.13 in Appendix-V. From Bhattacharyya (1985) it follows that the MMLEs from censored samples are also asymptotically as efficient as MLEs.

In small samples the performance of MMLEs between the two methods of modification is studied through Monte-Carlo simulation for selected values of $n, r$ (to the extent of $20 \%$ censoring in each chosen value of $n$ ). That is, the selected combinations are $n=5, r=4 ; n=10, r=8 ; n=15, r=12 ; n=20, r=16 ; n=25, r=20$. These are given in Table 3.14 respectively for methods - I and II. The variances of MMLEs from censored samples are found to be more than the corresponding variances of MMLEs from complete sample (a natural trend) as evidenced from the comparison between Table $(2.5,3.14)$ in Appendix VI. 


\section{Appendix - I}

Table 2.3: Slope $\left(\beta_{i}\right)$ and Intercept $\left(\alpha_{i}\right)$ in Modification to MLE by Method -I for $n=5,10,15,20$

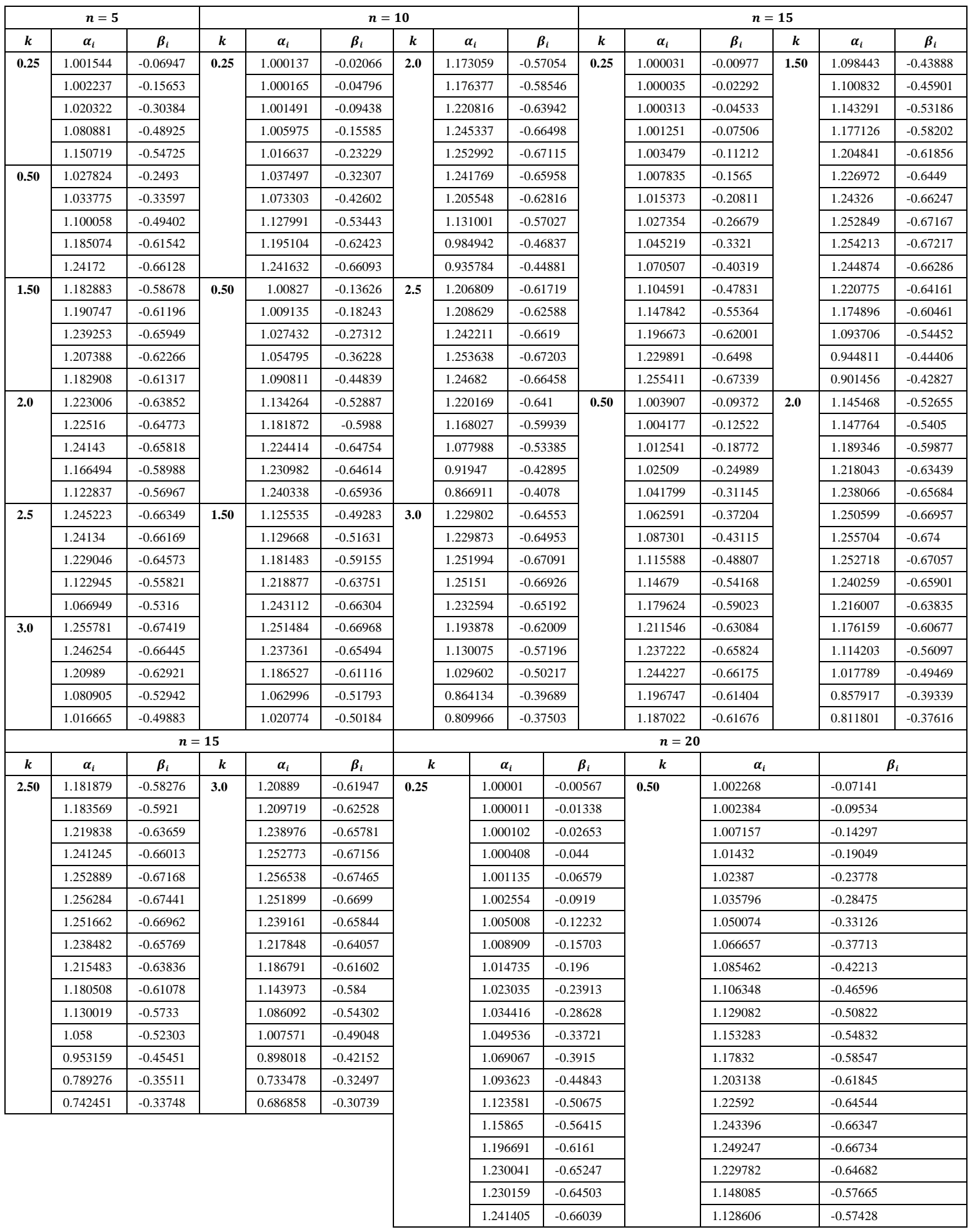




\section{Appendix - I (Continued)}

Table 2.3 (Continued): $\quad$ Slope $\left(\beta_{i}\right)$ and Intercept $\left(\alpha_{i}\right)$ in Modification to MLE by Method - I for $n=20,25$

\begin{tabular}{|c|c|c|c|c|c|c|c|c|c|c|c|c|c|c|c|c|c|}
\hline \multicolumn{12}{|c|}{$n=20$} & \multicolumn{6}{|c|}{$n=25$} \\
\hline$k$ & $\alpha_{i}$ & $\beta_{i}$ & $k$ & $\alpha_{i}$ & $\beta_{i}$ & $k$ & $\boldsymbol{\alpha}_{i}$ & $\beta_{i}$ & $k$ & $\alpha_{i}$ & $\beta_{i}$ & $k$ & $\boldsymbol{\alpha}_{i}$ & $\beta_{i}$ & $k$ & $\boldsymbol{\alpha}_{i}$ & $\beta_{i}$ \\
\hline \multirow[t]{20}{*}{1.50} & 1.082349 & -0.40267 & \multirow[t]{20}{*}{2.0} & 1.12777 & -0.4954 & \multirow[t]{20}{*}{2.5} & 1.164819 & -0.5571 & \multirow[t]{20}{*}{3.0} & 1.19359 & -0.59892 & \multirow[t]{25}{*}{0.25} & 1.00000 & -0.0037 & \multirow[t]{25}{*}{0.50} & 1.001479 & -0.05769 \\
\hline & 1.083927 & -0.42055 & & 1.129445 & -0.5083 & & 1.166204 & -0.5662 & & 1.19446 & -0.60511 & & 1.000000 & -0.0087 & & 1.00154 & -0.07698 \\
\hline & 1.119918 & $\begin{array}{l}-0.48957 \\
\end{array}$ & & 1.167338 & -0.5665 & & 1.201462 & -0.6132 & & 1.22501 & -0.64179 & & 1.00004 & -0.0174 & & 1.004622 & -0.11545 \\
\hline & 1.149383 & -0.53876 & & 1.194992 & -0.6045 & & 1.224602 & -0.6411 & & 1.24274 & -0.66125 & & 1.00017 & -0.0288 & & 1.009246 & -0.15387 \\
\hline & 1.17465 & -0.57661 & & 1.216345 & -0.6313 & & 1.240467 & -0.6588 & & 1.25289 & -0.67154 & & 1.00047 & -0.0432 & & 1.015413 & -0.19218 \\
\hline & 1.19651 & -0.60651 & & 1.232809 & -0.6504 & & 1.250798 & -0.6694 & & 1.25734 & -0.67562 & & 1.001066 & -0.0603 & & 1.023121 & -0.23035 \\
\hline & 1.215238 & -0.63015 & & 1.244968 & -0.6636 & & 1.256342 & -0.6747 & & 1.25690 & -0.67489 & & 1.00208 & -0.0803 & & 1.032364 & -0.26832 \\
\hline & 1.230836 & -0.648444 & & 1.253007 & -0.6716 & & 1.257376 & -0.6754 & & 1.25186 & -0.677007 & & 1.00371 & -0.1032 & & 1.043131 & -0.30601 \\
\hline & 1.2433116 & -0.66184 & & 1.256848 & -0.6751 & & 1.253888 & -0.6719 & & 1.24226 & -0.66151 & & 1.00614 & -0.1289 & & 1.055404 & -0.34335 \\
\hline & 1.251708 & -0.67052 & & 1.256201 & -0.6741 & & 1.245636 & -0.6643 & & 1.22789 & -0.64934 & & 1.009600 & -0.1574 & & 1.069153 & -0.38025 \\
\hline & 1.256041 & -0.67442 & & 1.250557 & -0.6687 & & 1.232164 & -0.6527 & & 1.20830 & -0.63349 & & 1.01435 & -0.1887 & & 1.08433 & -0.41658 \\
\hline & 1.255289 & -0.67322 & & 1.239151 & -0.6585 & & 1.21276 & -0.6368 & & 1.18285 & -0.61375 & & 1.02067 & -0.2227 & & 1.100865 & -0.45218 \\
\hline & 1.248272 & -0.6664 & & 1.220875 & -0.6430 & & 1.186396 & -0.6161 & & 1.15058 & -0.58972 & & 1.02887 & -0.2594 & & 1.11865 & -0.48687 \\
\hline & 1.233288 & -0.65306 & & 1.194131 & -0.6216 & & 1.1515855 & -0.5900 & & 1.11009 & -0.56078 & & 1.03929 & -0.2987 & & 1.137526 & -0.52038 \\
\hline & 1.207801 & -0.63184 & & 1.1565661 & -0.5931 & & 1.106143 & -0.5575 & & 1.05936 & -0.52601 & & 1.05228 & -0.3404 & & 1.157255 & -0.5524 \\
\hline & 1.167858 & -0.60063 & & 1.104525 & -0.5556 & & 1.0462727 & -0.5169 & & 0.9953 & -0.48398 & & 1.06819 & -0.3844 & & 1.177482 & -0.58246 \\
\hline & 1.106833 & -0.55591 & & 1.03201 & -0.5061 & & 0.96786 & -0.4657 & & 0.91288 & -0.43238 & & 1.08733 & -0.4301 & & 1.197669 & -0.609977 \\
\hline & 1.012267 & -0.49121 & & 0.927889 & -0.4392 & & 0.859507 & -0.3991 & & 0.80297 & -0.36708 & & 1.10996 & -0.4771 & & 1.216991 & -0.63407 \\
\hline & 0.854987 & -0.39175 & & 0.7665522 & -0.3427 & & 0.698763 & -0.3066 & & 0.64481 & -0.27875 & & 1.13612 & -0.5245 & & 1.2344142 & -0.65355 \\
\hline & 0.812791 & -0.37677 & & 0.723591 & -0.3272 & & 0.656338 & -0.2912 & & 0.60336 & -0.26369 & & 1.165428 & -0.5707 & & 1.24699 & -0.66655 \\
\hline \multicolumn{12}{|c|}{$n=25$} & & 1.19652 & -0.6133 & & 1.25187 & -0.67014 \\
\hline$k$ & $\alpha_{i}$ & $\beta_{i}$ & $k$ & $\boldsymbol{\alpha}_{i}$ & $\beta_{i}$ & $k$ & $\alpha_{i}$ & $\boldsymbol{\beta}_{i}$ & $k$ & $\alpha_{i}$ & $\beta_{i}$ & & 1.22569 & -0.6472 & & 1.241994 & -0.65932 \\
\hline \multirow[t]{25}{*}{1.50} & 1.071525 & -0.37604 & \multirow[t]{25}{*}{2.0} & 1.115236 & -0.4717 & \multirow[t]{25}{*}{2.50} & 1.152241 & -0.5370 & \multirow[t]{25}{*}{3.0} & 1.18188 & -0.58232 & & 1.24285 & -0.6621 & & 1.203239 & -0.62441 \\
\hline & 1.072659 & -0.39233 & & 1.116526 & -0.4838 & & 1.153386 & -0.5458 & & 1.18268 & -0.58855 & & 1.21551 & -0.6301 & & 1.098987 & -0.54209 \\
\hline & 1.104095 & -0.45789 & & 1.151321 & -0.5410 & & 1.1.877112 & -0.5935 & & 1.21311 & -0.62714 & & 1.21678 & -0.6399 & & 1.073867 & -0.53682 \\
\hline & 1.130135 & -0.50536 & & 1.1777333 & -0.5795 & & 1.210229 & -0.6233 & & 1.23217 & -0.64938 & & & & & & \\
\hline & 1.152864 & -0.5427 & & 1.198176 & -0.6077 & & 1.227257 & -0.6436 & & 1.24481 & -0.66315 & & & & & & \\
\hline & 1.173059 & -0.57314 & & 1.215216 & -0.6293 & & 1.239895 & -0.6578 & & 1.25288 & -0.6714 & & & & & & \\
\hline & 1.191077 & -0.59835 & & 1.229108 & -0.6458 & & 1.24896 & -0.6674 & & 1.25725 & -0.67558 & & & & & & \\
\hline & 1.207077 & -0.61929 & & 1.240186 & -0.6582 & & 1.254877 & -0.6733 & & 1.25840 & -0.67648 & & & & & & \\
\hline & 1.2211 & -0.63657 & & 1.248602 & -0.6671 & & 1.257854 & -0.6760 & & 1.25654 & -0.67457 & & & & & & \\
\hline & 1.2331 & -0.65052 & & 1.254384 & -0.6729 & & 1.25796 & -0.6759 & & 1.25178 & -0.67012 & & & & & & \\
\hline & 1.242959 & -0.66135 & & 1.257466 & -0.6757 & & 1.25516 & -0.6732 & & 1.24407 & -0.6633 & & & & & & \\
\hline & 1.250485 & -0.66916 & & 1.257695 & -0.6757 & & 1.249328 & -0.6678 & & 1.23333 & -0.65415 & & & & & & \\
\hline & 1.255405 & -0.67392 & & 1.254833 & -0.6728 & & 1.240249 & -0.6599 & & 1.21933 & -0.64267 & & & & & & \\
\hline & 1.257351 & -0.67553 & & 1.248542 & -0.6670 & & 1.227613 & -0.6492 & & 1.20181 & -0.62877 & & & & & & \\
\hline & 1.255834 & -0.67379 & & 1.238364 & -0.6581 & & 1.210996 & -0.6358 & & 1.18036 & -0.61231 & & & & & & \\
\hline & 1.250205 & -0.6684 & & 1.223689 & -0.6458 & & 1.189827 & -0.6192 & & 1.15445 & -0.59307 & & & & & & \\
\hline & 1.239589 & -0.6589 & & 1.203697 & -0.6297 & & 1.163345 & -0.5993 & & 1.12336 & -0.57073 & & & & & & \\
\hline & 1.222792 & -0.64466 & & 1.1177273 & -0.6093 & & 1.130518 & -0.5754 & & 1.08615 & -0.54486 & & & & & & \\
\hline & 1.198137 & -0.62479 & & 1.142868 & -0.5838 & & 1.089915 & -0.5470 & & 1.04148 & -0.51485 & & & & & & \\
\hline & 1.163179 & -0.59798 & & 1.098242 & -0.5521 & & 1.03449 & -0.5130 & & 0.98747 & -0.47983 & & & & & & \\
\hline & 1.114166 & -0.56227 & & 1.040004 & -0.5125 & & 0.976159 & -0.4720 & & 0.92130 & -0.43853 & & & & & & \\
\hline & 1.044902 & -0.5145 & & 0.962625 & -0.4624 & & 0.89494 & -0.4217 & & 0.83843 & -0.38893 & & & & & & \\
\hline & 0.943902 & -0.44892 & & 0.855998 & -0.3971 & & 0.786808 & -0.3580 & & 0.73069 & -0.32743 & & & & & & \\
\hline & 0.784629 & -0.35259 & & 0.697097 & -0.3057 & & 0.631357 & -0.2719 & & 0.57971 & -0.24602 & & & & & & \\
\hline & 0.74403 & -0.33841 & & 0.656918 & -0.2915 & & 0.592338 & -0.2580 & & 0.54205 & -0.23266 & & & & & & \\
\hline
\end{tabular}




\section{Appendix - II}

Table 2.4: $\quad$ Slope $\left(\beta_{i}\right)$ and Intercept $\left(\alpha_{i}\right)$ in Modification to MLE by Method -II for $n=5,10,15,20$

\begin{tabular}{|c|c|c|c|c|c|c|c|c|c|c|c|c|c|c|c|}
\hline \multicolumn{3}{|c|}{$n=5$} & \multicolumn{7}{|c|}{$n=10$} & \multicolumn{6}{|c|}{$n=15$} \\
\hline $\boldsymbol{k}$ & $\alpha_{i}$ & $\beta_{i}$ & $k$ & $\alpha_{i}$ & $\beta_{i}$ & $\boldsymbol{k}$ & $\alpha_{i}$ & & & $\boldsymbol{k}$ & $\alpha_{i}$ & $\beta_{i}$ & $\boldsymbol{k}$ & $\alpha_{i}$ & $\beta_{i}$ \\
\hline \multirow[t]{5}{*}{0.25} & 1.249164 & -0.66728 & \multirow[t]{10}{*}{0.25} & 1.369003 & -0.80108 & \multirow[t]{10}{*}{2.0} & 1.169044 & \multicolumn{2}{|c|}{-1.90112} & \multirow[t]{15}{*}{0.25} & 1.421925 & -0.87535 & \multirow[t]{15}{*}{1.50} & 1.225577 & -1.86011 \\
\hline & 1.032638 & -0.48712 & & 1.227534 & -0.64647 & & 1.310984 & -1. & & & 1.312165 & -0.73308 & & 1.349387 & -1.74887 \\
\hline & 0.837227 & -0.36099 & & 1.107134 & -0.543 & & 1.423283 & & 974 & & 1.219567 & -0.639 & & 1.435953 & -1.64161 \\
\hline & 0.638381 & -0.25422 & & 0.99642 & -0.46171 & & 1.502926 & & 928 & & 1.136041 & -0.56619 & & 1.495812 & -1.53547 \\
\hline & 0.408803 & -0.14952 & & 0.889993 & -0.39261 & & 1.546091 & -1. & & & 1.057889 & -0.50548 & & 1.533399 & -1.42939 \\
\hline \multirow[t]{5}{*}{0.50} & 1.497945 & -1.01809 & & 0.784217 & -0.33074 & & 1.547629 & & 121 & & 0.982965 & -0.45254 & & 1.550882 & -1.32281 \\
\hline & 1.33462 & -0.75879 & & 0.675774 & -0.27299 & & 1.50011 & $-1 .($ & 333 & & 0.9098 & -0.4049 & & 1.54924 & -1.21534 \\
\hline & 1.136041 & -0.56619 & & 0.560626 & -0.21685 & & 1.391787 & -0.8 & 146 & & 0.837227 & -0.36099 & & 1.528641 & -1.10668 \\
\hline & 0.901726 & -0.39986 & & 0.432266 & -0.15948 & & 1.201172 & -0 & 214 & & 0.764192 & -0.31967 & & 1.488557 & -0.99648 \\
\hline & 0.603306 & -0.2371 & & 0.275998 & -0.09586 & & 0.876108 & -0 & 413 & & 0.6896 & -0.28007 & & 1.427703 & -0.88435 \\
\hline \multirow[t]{5}{*}{1.50} & 1.410392 & -1.67716 & \multirow[t]{10}{*}{0.50} & 1.546728 & -1.19433 & \multirow[t]{10}{*}{2.5} & 1.096036 & -1.9 & 764 & & 0.612156 & -0.24138 & & 1.343793 & -0.7697 \\
\hline & 1.541392 & -1.39394 & & 1.48529 & -0.98934 & & 1.215465 & & 781 & & 0.530129 & -0.20276 & & 1.232961 & -0.65162 \\
\hline & 1.528641 & -1.10668 & & 1.399023 & -0.84158 & & 1.331993 & & 681 & & 0.440875 & -0.16318 & & 1.088414 & -0.52846 \\
\hline & 1.374521 & -0.80824 & & 1.300797 & -0.72057 & & 1.433348 & & 543 & & 0.339641 & -0.12101 & & 0.896671 & -0.39672 \\
\hline & 1.030754 & -0.48577 & & 1.19328 & -0.61506 & & 1.509276 & & 373 & & 0.215309 & -0.07281 & & 0.622995 & -0.24665 \\
\hline 2.0 & 1.289313 & -1.80725 & & 1.07626 & -0.5192 & & 1.549232 & & 139 & 0.50 & 1.552458 & -1.28535 & 2.0 & 1.119018 & -1.93369 \\
\hline & 1.480221 & -1.56749 & & 0.947751 & -0.42915 & & 1.540639 & & & & 1.528641 & -1.10668 & & 1.225577 & -1.86011 \\
\hline & 1.552458 & -1.28535 & & 0.803547 & -0.3416 & & 1.46619 & & 071 & & 1.480384 & -0.97894 & & 1.318899 & -1.77972 \\
\hline & 1.471532 & -0.96104 & & 0.63494 & -0.25252 & & 1.29738 & & 686 & & 1.421925 & -0.87535 & & 1.398141 & -1.69287 \\
\hline & 1.158678 & -0.58502 & & 0.419563 & -0.15407 & & 0.970534 & & 418 & & 1.357266 & -0.7862 & & 1.462347 & -1.59984 \\
\hline 2.5 & 1.195363 & -1.88263 & 1.50 & 1.287698 & -1.80869 & 3.0 & 1.053683 & & 185 & & 1.287851 & -0.70668 & & 1.510406 & -1.50084 \\
\hline & 1.401892 & -1.68814 & & 1.429281 & -1.65129 & & 1.145805 & & 664 & & 1.214131 & -0.63397 & & 1.540989 & -1.396 \\
\hline & 1.534466 & -1.42518 & & 1.51191 & -1.49693 & & 1.252964 & & 836 & & 1.136041 & -0.56619 & & 1.552458 & -1.28535 \\
\hline & 1.524217 & -1.09133 & & 1.549142 & -1.34224 & & 1.36101 & & 634 & & 1.053129 & -0.50198 & & 1.542733 & -1.16885 \\
\hline & 1.25667 & -0.6747 & & 1.545511 & -1.18584 & & 1.456822 & & & & 0.964558 & -0.4402 & & 1.509074 & -1.04629 \\
\hline 3.0 & 1.12898 & -1.92745 & & 1.50148 & -1.02671 & & 1.526279 & & 485 & & 0.868973 & -0.37982 & & 1.447701 & -0.91727 \\
\hline & 1.325701 & -1.77307 & & 1.414249 & -0.86371 & & 1.552454 & & 142 & & 0.764192 & -0.31967 & & 1.353059 & -0.78099 \\
\hline & 1.495812 & -1.53547 & & 1.276719 & -0.69504 & & 1.51257 & & 1589 & & 0.646485 & -0.25824 & & 1.216186 & -0.63586 \\
\hline & 1.547891 & -1.20333 & & 1.073224 & -0.51691 & & 1.370622 & & 317 & & 0.508628 & -0.193 & & 1.020295 & -0.47834 \\
\hline & 1.332899 & -0.75677 & & 0.761866 & -0.3184 & & 1.050424 & & 1999 & & 0.332728 & -0.11823 & & 0.723621 & -0.29783 \\
\hline & & & & & & & & & & & $n=2$ & & & & \\
\hline $\boldsymbol{k}$ & $\alpha_{i}$ & $\boldsymbol{\beta}_{i}$ & $k$ & $\alpha_{i}$ & $\boldsymbol{\beta}_{i}$ & t & & & & & $\boldsymbol{k}$ & $\alpha_{i}$ & & & \\
\hline 2.50 & 1.06102 & -1.96779 & 3.0 & 1.030882 & -1.98413 & 0.25 & & & -0.9 & & 0.50 & 1.548926 & & -1.34425 & \\
\hline & 1.140158 & -1.92031 & & 1.085415 & -1.95389 & & & & -0.7 & & & 1.544948 & & -1.18215 & \\
\hline & 1.222981 & -1.86211 & & 1.152223 & -1.91243 & & & & -0.7 & & & 1.516351 & & -1.06675 & \\
\hline & 1.303864 & -1.79398 & & 1.225577 & -1.86011 & & & & -0.6 & & & 1.477783 & & -0.97357 & \\
\hline & 1.37882 & -1.7162 & & 1.300894 & -1.79673 & & & & -0.5 & & & 1.433608 & & -0.89377 & \\
\hline & 1.444436 & -1.62883 & & 1.373929 & -1.72185 & & & & -0.5 & & & 1.385612 & & -0.82301 & \\
\hline & 1.497445 & -1.53186 & & 1.440413 & -1.63496 & & & & -0.4 & & & 1.33462 & & -0.75879 & \\
\hline & 1.534466 & -1.42518 & & 1.495812 & -1.53547 & & & 941 & -0.4 & & & 1.281013 & & -0.6995 & \\
\hline & 1.551754 & -1.3086 & & 1.535065 & -1.42275 & & & & -0.4 & & & 1.224924 & & -0.64401 & \\
\hline & 1.544892 & -1.18179 & & 1.552245 & -1.29604 & & & 872 & -0.3 & & & 1.166326 & & -0.59152 & \\
\hline & 1.508304 & -1.04423 & & 1.540003 & -1.15438 & & & 1516 & -0.3 & & & 1.10506 & & -0.54137 & \\
\hline & 1.434376 & -0.89501 & & 1.488557 & -0.99648 & & & 659 & -0.3 & & & 1.040848 & & -0.49303 & \\
\hline & 1.31156 & -0.73241 & & 1.383542 & -0.82022 & & & 804 & -0.2 & & & 0.973269 & & -0.44601 & \\
\hline & 1.119332 & -0.55267 & & 1.20035 & -0.6214 & & & 381 & -0.2 & & & 0.901726 & & -0.39986 & \\
\hline & 0.808757 & -0.34456 & & 0.882557 & -0.38805 & & & 688 & -0.2 & & & 0.825363 & & -0.35409 & \\
\hline & & & & & & & & & -0.1 & & & 0.742922 & & -0.30813 & \\
\hline & & & & & & & & 346 & -0.1 & & & 0.652468 & & -0.26123 & \\
\hline & & & & & & & & 226 & -0.1 & & & 0.550762 & & -0.21226 & \\
\hline & & & & & & & & 545 & -0.0 & & & 0.431637 & & -0.15921 & \\
\hline & & & & & & & & & -0.0 & & & 0.280087 & & -0.09744 & \\
\hline
\end{tabular}


Appendix - II (Continued)

Table 2.4 (Continued): $\quad$ Slope $\left(\beta_{i}\right)$ and Intercept $\left(\alpha_{i}\right)$ in Modification to MLE by Method -II for $n=20,25$

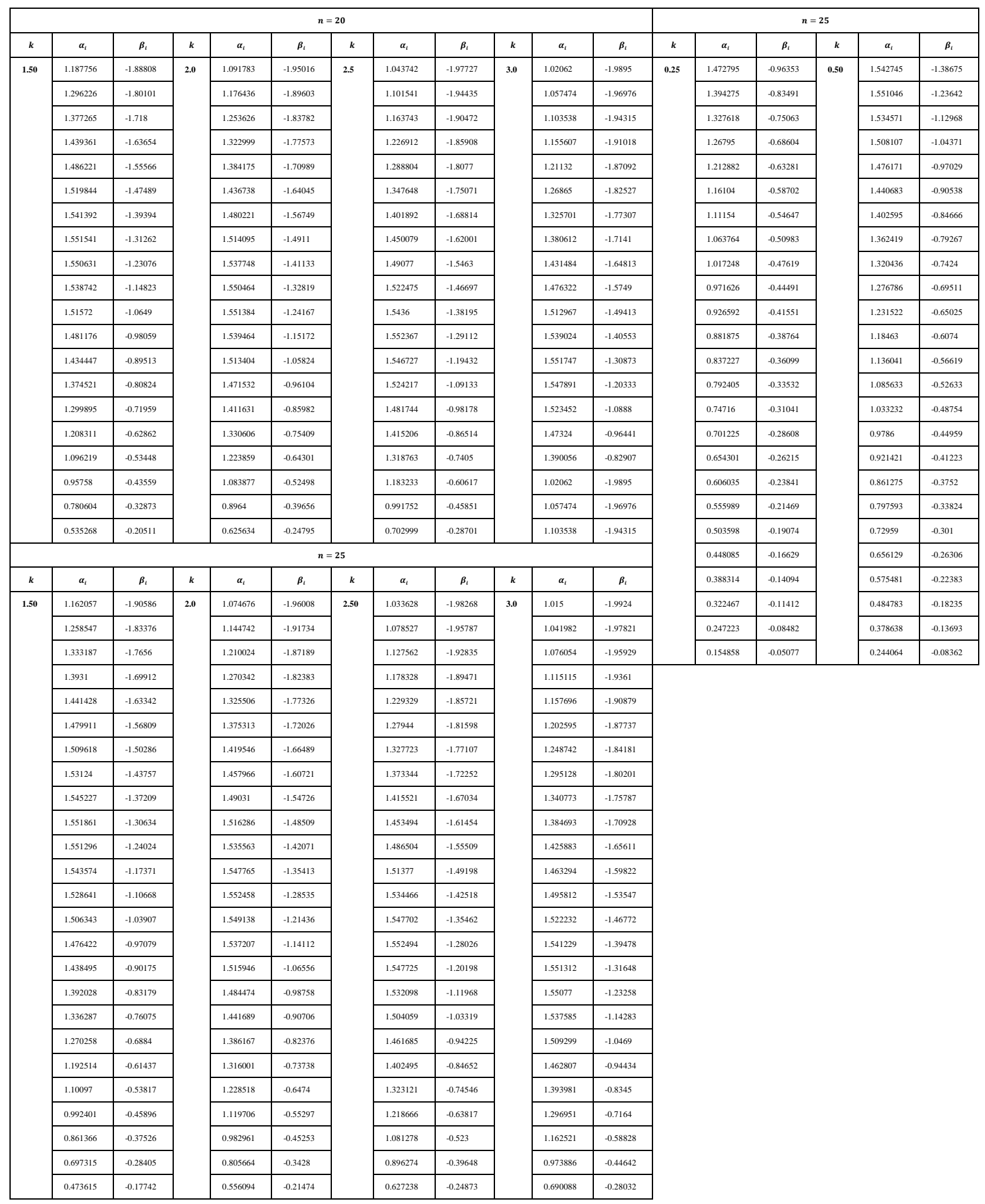




\section{Appendix-III}

Table 2.5: Empirical Variances of $\hat{\lambda}$ based on MMLE: Method -I \& Method -II

\begin{tabular}{|c|c|c|c|c|c|c|}
\hline \multicolumn{7}{|c|}{ MMLE: Method -I } \\
\hline$n$ & 0.25 & 0.50 & $\mathbf{1 . 5 0}$ & 2.0 & 2.5 & 3.0 \\
\hline & & & & & & \\
\hline 5 & 0.1341425 & 0.097691 & 0.044699 & 0.035495 & 0.02685 & 0.02321 \\
\hline 10 & 0.099113 & 0.060441 & 0.020486 & 0.016022 & 0.012314 & 0.010444 \\
\hline 15 & 0.07457 & 0.038852 & 0.013336 & 0.010474 & 0.00819 & 0.006875 \\
\hline 20 & 0.059105 & 0.028732 & 0.009685 & 0.00823 & 0.005912 & 0.005017 \\
\hline 25 & 0.0469 & 0.021249 & 0.007989 & 0.006167 & 0.004862 & 0.004187 \\
\hline \multicolumn{7}{|c|}{ MMLE: Method -II } \\
\hline \multirow{2}{*}{$n$} & 0.25 & 0.50 & 1.50 & 2.0 & 2.5 & 3.0 \\
\hline & & & & & & \\
\hline 5 & 0.130502 & 0.099553 & 0.040283 & 0.028477 & 0.019177 & 0.013697 \\
\hline 10 & 0.097346 & 0.057394 & 0.018313 & 0.012291 & 0.008974 & 0.00647 \\
\hline 15 & 0.079233 & 0.038871 & 0.011892 & 0.007887 & 0.005866 & 0.004383 \\
\hline 20 & 0.064955 & 0.028415 & 0.008615 & 0.006064 & 0.00426 & 0.003162 \\
\hline 25 & 0.053599 & 0.020655 & 0.006947 & 0.00485 & 0.003434 & 0.0025 \\
\hline
\end{tabular}

Table 3.1: Correlation Coefficient between $(\mathrm{z}, \mathrm{h}(\mathrm{z}))$

\begin{tabular}{|c|c|c|c|c|c|c|c|c|}
\hline \multirow[t]{2}{*}{$n$} & \multirow[b]{2}{*}{$r$} & $\mathrm{k}=3$ & \multirow[t]{2}{*}{$n$} & \multirow[b]{2}{*}{$r$} & $k=3$ & \multirow[t]{2}{*}{$n$} & \multirow[b]{2}{*}{$r$} & $\mathrm{k}=\mathbf{3}$ \\
\hline & & $\begin{array}{l}\text { Correlation } \\
\text { Coefficient }\end{array}$ & & & $\begin{array}{l}\text { Correlation } \\
\text { Coefficient }\end{array}$ & & & $\begin{array}{l}\text { Correlation } \\
\text { Coefficient }\end{array}$ \\
\hline \multirow{3}{*}{5} & 2 & 0.998093 & \multirow{24}{*}{20} & 2 & 0.995589 & \multirow{24}{*}{25} & 2 & 0.995188 \\
\hline & 3 & 0.998973 & & 3 & 0.997319 & & 3 & 0.997026 \\
\hline & 4 & 0.999855 & & 4 & 0.998184 & & 4 & 0.997953 \\
\hline \multirow{8}{*}{10} & 2 & 0.996872 & & 5 & 0.998689 & & 5 & 0.998499 \\
\hline & 3 & 0.998236 & & 6 & 0.999013 & & 6 & 0.998853 \\
\hline & 4 & 0.998887 & & 7 & 0.999234 & & 7 & 0.999096 \\
\hline & 5 & 0.999246 & & 8 & 0.999392 & & 8 & 0.999272 \\
\hline & 6 & 0.999457 & & 9 & 0.999509 & & 9 & 0.999403 \\
\hline & 7 & 0.999574 & & 10 & 0.999597 & & 10 & 0.999504 \\
\hline & 8 & 0.999587 & & 11 & 0.999664 & & 11 & 0.999583 \\
\hline & 9 & 0.999936 & & 12 & 0.999717 & & 12 & 0.999646 \\
\hline \multirow{13}{*}{15} & 2 & 0.996117 & & 13 & 0.999757 & & 13 & 0.999697 \\
\hline & 3 & 0.997702 & & 14 & 0.999786 & & 14 & 0.999738 \\
\hline & 4 & 0.998482 & & 15 & 0.999806 & & 15 & 0.999771 \\
\hline & 5 & 0.99893 & & 16 & 0.999814 & & 16 & 0.999798 \\
\hline & 6 & 0.999213 & & 17 & 0.999802 & & 17 & 0.99982 \\
\hline & 7 & 0.999403 & & 18 & 0.999734 & & 18 & 0.999836 \\
\hline & 8 & 0.999535 & & 19 & 0.999958 & & 19 & 0.999848 \\
\hline & 9 & 0.999628 & & & & & 20 & 0.999853 \\
\hline & 10 & 0.999693 & & & & & 21 & 0.99985 \\
\hline & 11 & 0.999733 & & & & & 22 & 0.999829 \\
\hline & 12 & 0.999745 & & & & & 23 & 0.999755 \\
\hline & 13 & 0.999693 & & & & & 24 & 0.999961 \\
\hline & 14 & 0.999951 & & & & & & \\
\hline
\end{tabular}


Appendix - IV

Table 3.2: $\quad$ Slope $\left(\delta_{r}\right)$ and Intercept $\left(\Upsilon_{r}\right)$ in Modification to MLE from Censored Sample by Method $-\mathrm{I}: \mathrm{k}=\mathbf{0 . 2 5}$

\begin{tabular}{|c|c|c|c|c|c|c|c|c|c|}
\hline $\boldsymbol{k}$ & $n$ & $r$ & $\delta_{r}$ & $\mathbf{Y}_{r}$ & $\boldsymbol{k}$ & $n$ & $r$ & $\delta_{r}$ & $\mathbf{Y}_{r}$ \\
\hline \multirow{42}{*}{0.25} & \multirow[t]{3}{*}{5} & 2 & 3.473945 & 0.087458 & \multirow{23}{*}{0.25} & \multirow[t]{23}{*}{25} & 2 & 7.652104 & 0.019929 \\
\hline & & 3 & 3.758664 & 0.093293 & & & 3 & 5.561148 & 0.03954 \\
\hline & & 4 & 4.879543 & -0.2058 & & & 4 & 4.560551 & 0.058519 \\
\hline & \multirow{8}{*}{10} & 2 & 4.171105 & 0.048719 & & & 5 & 4.000841 & 0.076474 \\
\hline & & 3 & 3.504577 & 0.090939 & & & 6 & 3.665968 & 0.092919 \\
\hline & & 4 & 3.354078 & 0.117855 & & & 7 & 3.463801 & 0.107262 \\
\hline & & 5 & 3.463016 & 0.114755 & & & 8 & 3.348766 & 0.118779 \\
\hline & & 6 & 3.769879 & 0.055961 & & & 9 & 3.296087 & 0.126585 \\
\hline & & 7 & 4.289639 & -0.10754 & & & 10 & 3.291509 & 0.129589 \\
\hline & & 8 & 5.115904 & -0.48441 & & & 11 & 3.326662 & 0.126444 \\
\hline & & 9 & 6.551342 & -1.39176 & & & 12 & 3.396789 & 0.115459 \\
\hline & \multirow[t]{13}{*}{15} & 2 & 5.265932 & 0.032981 & & & 13 & 3.499593 & 0.094491 \\
\hline & & 3 & 4.07629 & 0.064297 & & & 14 & 3.634655 & 0.060776 \\
\hline & & 4 & 3.574889 & 0.092048 & & & 15 & 3.803218 & 0.010684 \\
\hline & & 5 & 3.365154 & 0.113558 & & & 16 & 4.008237 & -0.06067 \\
\hline & & 6 & 3.318288 & 0.125085 & & & 17 & 4.254695 & -0.16001 \\
\hline & & 7 & 3.383293 & 0.121334 & & & 18 & 4.550294 & -0.29696 \\
\hline & & 8 & 3.540161 & 0.094621 & & & 19 & 4.906744 & -0.48575 \\
\hline & & 9 & 3.785417 & 0.033383 & & & 20 & 5.342221 & -0.7486 \\
\hline & & 10 & 4.128378 & -0.08071 & & & 21 & 5.886358 & -1.12252 \\
\hline & & 11 & 4.593546 & -0.27881 & & & 22 & 6.591664 & -1.67524 \\
\hline & & 12 & 5.231281 & -0.6192 & & & 23 & 7.565199 & -2.54986 \\
\hline & & 13 & 6.150807 & -1.22836 & & & 24 & 9.095302 & -4.1429 \\
\hline & & 14 & 7.655368 & -2.466 & & & & & \\
\hline & \multirow{18}{*}{20} & 2 & 6.443683 & 0.024857 & & & & & \\
\hline & & 3 & 4.793209 & 0.049064 & & & & & \\
\hline & & 4 & 4.029089 & 0.071939 & & & & & \\
\hline & & 5 & 3.627491 & 0.092594 & & & & & \\
\hline & & 6 & 3.414842 & 0.109882 & & & & & \\
\hline & & 7 & 3.317896 & 0.122332 & & & & & \\
\hline & & 8 & 3.301345 & 0.128043 & & & & & \\
\hline & & 9 & 3.347123 & 0.124542 & & & & & \\
\hline & & 10 & 3.44629 & 0.108555 & & & & & \\
\hline & & 11 & 3.595567 & 0.075658 & & & & & \\
\hline & & 12 & 3.795938 & 0.019719 & & & & & \\
\hline & & 13 & 4.052448 & -0.06805 & & & & & \\
\hline & & 14 & 4.375019 & -0.20077 & & & & & \\
\hline & & 15 & 4.780662 & -0.39905 & & & & & \\
\hline & & 16 & 5.298423 & -0.69753 & & & & & \\
\hline & & 17 & 5.981007 & -1.16021 & & & & & \\
\hline & & 18 & 6.937358 & -1.92385 & & & & & \\
\hline & & 19 & 8.463337 & -3.37119 & & & & & \\
\hline
\end{tabular}


Table 3.3: $\quad$ Slope $\left(\boldsymbol{\delta}_{r}\right)$ and Intercept $\left(\Upsilon_{r}\right)$ in Modification to MLE from Censored Sample by Method $-I: k=0.50$

\begin{tabular}{|c|c|c|c|c|c|c|c|c|c|}
\hline $\boldsymbol{k}$ & $n$ & $r$ & $\delta_{r}$ & $\mathbf{Y}_{r}$ & $\boldsymbol{k}$ & $n$ & $r$ & $\boldsymbol{\delta}_{r}$ & $\mathbf{r}_{r}$ \\
\hline \multirow{42}{*}{0.50} & \multirow[t]{3}{*}{5} & 2 & 1.826788 & -0.08609 & \multirow[t]{23}{*}{0.50} & \multirow[t]{23}{*}{25} & 2 & 1.159598 & -0.00321 \\
\hline & & 3 & 2.415824 & -0.31404 & & & 3 & 1.243444 & -0.00985 \\
\hline & & 4 & 3.289925 & -0.8439 & & & 4 & 1.330307 & -0.02019 \\
\hline & \multirow{8}{*}{10} & 2 & 1.400954 & -0.02038 & & & 5 & 1.420469 & -0.03454 \\
\hline & & 3 & 1.631698 & -0.06555 & & & 6 & 1.514261 & -0.05325 \\
\hline & & 4 & 1.890025 & -0.14219 & & & 7 & 1.612065 & -0.07677 \\
\hline & & 5 & 2.185062 & -0.26078 & & & 8 & 1.714327 & -0.10559 \\
\hline & & 6 & 2.531458 & -0.43886 & & & 9 & 1.821572 & -0.14035 \\
\hline & & 7 & 2.954923 & -0.70845 & & & 10 & 1.934425 & -0.18178 \\
\hline & & 8 & 3.507629 & -1.13701 & & & 11 & 2.053629 & -0.23079 \\
\hline & & 9 & 4.330148 & -1.91053 & & & 12 & 2.180088 & -0.28852 \\
\hline & \multirow[t]{13}{*}{15} & 2 & 1.266246 & -0.00896 & & & 13 & 2.314904 & -0.35637 \\
\hline & & 3 & 1.411523 & -0.02804 & & & 14 & 2.45945 & -0.43611 \\
\hline & & 4 & 1.566639 & -0.05873 & & & 15 & 2.61546 & -0.53007 \\
\hline & & 5 & 1.733364 & -0.10301 & & & 16 & 2.785168 & -0.64126 \\
\hline & & 6 & 1.914005 & -0.16355 & & & 17 & 2.971528 & -0.77378 \\
\hline & & 7 & 2.111638 & -0.244 & & & 18 & 3.178554 & -0.93333 \\
\hline & & 8 & 2.330478 & -0.34953 & & & 19 & 3.411915 & -1.12813 \\
\hline & & 9 & 2.576509 & -0.48772 & & & 20 & 3.680009 & -1.37068 \\
\hline & & 10 & 2.858645 & -0.67022 & & & 21 & 3.996102 & -1.68119 \\
\hline & & 11 & 3.191033 & -0.91612 & & & 22 & 4.383204 & -2.0956 \\
\hline & & 12 & 3.598311 & -1.25975 & & & 23 & 4.887602 & -2.68785 \\
\hline & & 13 & 4.130188 & -1.7724 & & & 24 & 5.633568 & -3.659 \\
\hline & & 14 & 4.920114 & -2.64884 & & & & & \\
\hline & \multirow[t]{18}{*}{20} & 2 & 1.19952 & -0.00502 & & & & & \\
\hline & & 3 & 1.305791 & -0.01553 & & & & & \\
\hline & & 4 & 1.417061 & -0.03206 & & & & & \\
\hline & & 5 & 1.533948 & -0.05531 & & & & & \\
\hline & & 6 & 1.657199 & -0.08611 & & & & & \\
\hline & & 7 & 1.787721 & -0.12551 & & & & & \\
\hline & & 8 & 1.926631 & -0.17482 & & & & & \\
\hline & & 9 & 2.075322 & -0.23572 & & & & & \\
\hline & & 10 & 2.235557 & -0.31042 & & & & & \\
\hline & & 11 & 2.409619 & -0.40181 & & & & & \\
\hline & & 12 & 2.600527 & -0.51382 & & & & & \\
\hline & & 13 & 2.812398 & -0.65197 & & & & & \\
\hline & & 14 & 3.051053 & -0.82425 & & & & & \\
\hline & & 15 & 3.325129 & -1.04284 & & & & & \\
\hline & & 16 & 3.648297 & -1.32758 & & & & & \\
\hline & & 17 & 4.0443 & -1.71384 & & & & & \\
\hline & & 18 & 4.560933 & -2.27466 & & & & & \\
\hline & & 19 & 5.326456 & -3.20889 & & & & & \\
\hline
\end{tabular}


Table 3.4: $\quad$ Slope $\left(\delta_{r}\right)$ and Intercept $\left(\Upsilon_{r}\right)$ in Modification to MLE from Censored Sample by Method $-\mathrm{I}: \mathrm{k}=\mathbf{1 . 5 0}$

\begin{tabular}{|c|c|c|c|c|c|c|c|c|c|}
\hline $\boldsymbol{k}$ & $n$ & $r$ & $\boldsymbol{\delta}_{r}$ & $\mathbf{Y}_{r}$ & $\boldsymbol{k}$ & $n$ & $r$ & $\delta_{r}$ & $\mathbf{Y}_{r}$ \\
\hline \multirow{42}{*}{1.50} & \multirow[t]{3}{*}{5} & 2 & 1.037595 & -0.43145 & \multirow[t]{23}{*}{1.50} & \multirow[t]{23}{*}{25} & 2 & 0.418654 & -0.09955 \\
\hline & & 3 & 1.390186 & -0.74688 & & & 3 & 0.554401 & -0.16364 \\
\hline & & 4 & 1.752863 & -1.143 & & & 4 & 0.666795 & -0.22453 \\
\hline & \multirow{8}{*}{10} & 2 & 0.705847 & -0.22912 & & & 5 & 0.765727 & -0.28406 \\
\hline & & 3 & 0.929575 & -0.37878 & & & 6 & 0.855712 & -0.34306 \\
\hline & & 4 & 1.120058 & -0.52797 & & & 7 & 0.939347 & -0.40208 \\
\hline & & 5 & 1.29607 & -0.68457 & & & 8 & 1.018326 & -0.46155 \\
\hline & & 6 & 1.468208 & -0.85582 & & & 9 & 1.093854 & -0.52187 \\
\hline & & 7 & 1.646176 & -1.05229 & & & 10 & 1.16685 & -0.58341 \\
\hline & & 8 & 1.84409 & -1.29439 & & & 11 & 1.238062 & -0.64656 \\
\hline & & 9 & 2.096124 & -1.63751 & & & 12 & 1.308135 & -0.71176 \\
\hline & \multirow[t]{13}{*}{15} & 2 & 0.561846 & -0.15872 & & & 13 & 1.377662 & -0.77948 \\
\hline & & 3 & 0.740641 & -0.26088 & & & 14 & 1.447222 & -0.85033 \\
\hline & & 4 & 0.889665 & -0.35945 & & & 15 & 1.51742 & -0.925 \\
\hline & & 5 & 1.022675 & -0.45807 & & & 16 & 1.588929 & -1.00441 \\
\hline & & 6 & 1.146155 & -0.55883 & & & 17 & 1.662549 & -1.08973 \\
\hline & & 7 & 1.264116 & -0.66359 & & & 18 & 1.739287 & -1.1826 \\
\hline & & 8 & 1.37957 & -0.77438 & & & 19 & 1.820503 & -1.28532 \\
\hline & & 9 & 1.495225 & -0.89375 & & & 20 & 1.90816 & -1.40135 \\
\hline & & 10 & 1.614016 & -1.02532 & & & 21 & 2.005341 & -1.53631 \\
\hline & & 11 & 1.739774 & -1.17472 & & & 22 & 2.117433 & -1.70024 \\
\hline & & 12 & 1.1878524 & -1.35181 & & & 23 & 2.255529 & -1.9141 \\
\hline & & 13 & 2.042019 & -1.57693 & & & 24 & 2.450671 & -2.23697 \\
\hline & & 14 & 2.262397 & -1.90695 & & & & & \\
\hline & \multirow[t]{18}{*}{20} & 2 & 0.476536 & -0.12214 & & & & & \\
\hline & & 3 & 0.62963 & -0.20063 & & & & & \\
\hline & & 4 & 0.756544 & -0.27555 & & & & & \\
\hline & & 5 & 0.868683 & -0.34931 & & & & & \\
\hline & & 6 & 0.971285 & -0.42312 & & & & & \\
\hline & & 7 & 1.067408 & -0.49783 & & & & & \\
\hline & & 8 & 1.1591 & -0.57419 & & & & & \\
\hline & & 9 & 1.247893 & -0.65298 & & & & & \\
\hline & & 10 & 1.335048 & -0.73503 & & & & & \\
\hline & & 11 & 1.42171 & -0.82134 & & & & & \\
\hline & & 12 & 1.509028 & -0.91318 & & & & & \\
\hline & & 13 & 1.598284 & -1.01219 & & & & & \\
\hline & & 14 & 1.691053 & -1.12073 & & & & & \\
\hline & & 15 & 1.789478 & -1.24225 & & & & & \\
\hline & & 16 & 1.8968 & -1.38234 & & & & & \\
\hline & & 17 & 2.018568 & -1.55092 & & & & & \\
\hline & & 18 & 2.166078 & -1.76865 & & & & & \\
\hline & & 19 & 2.370786 & -2.09366 & & & & & \\
\hline
\end{tabular}


Table 3.5: $\quad$ Slope $\left(\boldsymbol{\delta}_{r}\right)$ and Intercept $\left(\Upsilon_{r}\right)$ in Modification to MLE from Censored Sample by Method -I: $k=2.0$

\begin{tabular}{|c|c|c|c|c|c|c|c|c|c|}
\hline $\boldsymbol{k}$ & $n$ & $r$ & $\delta_{r}$ & $\mathbf{Y}_{r}$ & $\boldsymbol{k}$ & $n$ & $r$ & $\boldsymbol{\delta}_{r}$ & $\mathbf{Y}_{r}$ \\
\hline \multirow{42}{*}{2.0} & \multirow[t]{3}{*}{5} & 2 & 0.91197 & -0.48689 & \multirow[t]{23}{*}{2.0} & \multirow[t]{23}{*}{25} & 2 & 0.370266 & -0.12903 \\
\hline & & 3 & 1.197436 & -0.77718 & & & 3 & 0.495 & -0.20352 \\
\hline & & 4 & 1.467311 & -1.10273 & & & 4 & 0.596585 & -0.2713 \\
\hline & \multirow{8}{*}{10} & 2 & 0.628161 & -0.27758 & & & 5 & 0.684557 & -0.33524 \\
\hline & & 3 & 0.824474 & -0.43362 & & & 6 & 0.763285 & -0.39663 \\
\hline & & 4 & 0.984712 & -0.57881 & & & 7 & 0.835287 & -0.4563 \\
\hline & & 5 & 1.126877 & -0.72222 & & & 8 & 0.902198 & -0.51483 \\
\hline & & 6 & 1.260369 & -0.87035 & & & 9 & 0.965167 & -0.57269 \\
\hline & & 7 & 1.392817 & -1.03116 & & & 10 & 1.025057 & -0.63029 \\
\hline & & 8 & 1.534198 & -1.21889 & & & 11 & 1.082551 & -0.688 \\
\hline & & 9 & 1.707694 & -1.47203 & & & 12 & 1.138216 & -0.74622 \\
\hline & \multirow[t]{13}{*}{15} & 2 & 0.499965 & -0.19869 & & & 13 & 1.192555 & -0.80532 \\
\hline & & 3 & 0.661061 & -0.31108 & & & 14 & 1.246035 & -0.86576 \\
\hline & & 4 & 0.79155 & -0.41369 & & & 15 & 1.29912 & -0.92805 \\
\hline & & 5 & 0.904879 & -0.51165 & & & 16 & 1.352304 & -0.99283 \\
\hline & & 6 & 1.007294 & -0.60758 & & & 17 & 1.406152 & -1.06092 \\
\hline & & 7 & 1.102542 & -0.7034 & & & 18 & 1.461356 & -1.13342 \\
\hline & & 8 & 1.193281 & -0.80092 & & & 19 & 1.518835 & -1.21189 \\
\hline & & 9 & 1.281731 & -0.90213 & & & 20 & 1.579904 & -1.2987 \\
\hline & & 10 & 1.370112 & -1.00963 & & & 21 & 1.646627 & -1.39769 \\
\hline & & 11 & 1.46113 & -1.12732 & & & 22 & 1.722641 & -1.5158 \\
\hline & & 12 & 1.558884 & -1.26195 & & & 23 & 1.81552 & -1.66773 \\
\hline & & 13 & 1.671285 & -1.42752 & & & 24 & 1.946813 & -1.89572 \\
\hline & & 14 & 1.820208 & -1.66392 & & & & & \\
\hline & \multirow[t]{18}{*}{20} & 2 & 0.422909 & -0.15604 & & & & & \\
\hline & & 3 & 0.562606 & -0.24524 & & & & & \\
\hline & & 4 & 0.675977 & -0.32639 & & & & & \\
\hline & & 5 & 0.77411 & -0.40316 & & & & & \\
\hline & & 6 & 0.862097 & -0.47728 & & & & & \\
\hline & & 7 & 0.942886 & -0.54987 & & & & & \\
\hline & & 8 & 1.01842 & -0.62179 & & & & & \\
\hline & & 9 & 1.09011 & -0.69382 & & & & & \\
\hline & & 10 & 1.159067 & -0.76669 & & & & & \\
\hline & & 11 & 1.22625 & -0.84119 & & & & & \\
\hline & & 12 & 1.292562 & -0.91827 & & & & & \\
\hline & & 13 & 1.358955 & -0.99909 & & & & & \\
\hline & & 14 & 1.426541 & -1.08528 & & & & & \\
\hline & & 15 & 1.496786 & -1.1792 & & & & & \\
\hline & & 16 & 1.571871 & -1.28465 & & & & & \\
\hline & & 17 & 1.655519 & -1.40842 & & & & & \\
\hline & & 18 & 1.755375 & -1.56488 & & & & & \\
\hline & & 19 & 1.893085 & -1.79525 & & & & & \\
\hline
\end{tabular}


Table 3.6: Slope $\left(\delta_{r}\right)$ and Intercept $\left(\Upsilon_{r}\right)$ in Modification to MLE from Censored Sample by Method $-\mathrm{I}: \mathrm{k}=\mathbf{2 . 5}$

\begin{tabular}{|c|c|c|c|c|c|c|c|c|c|}
\hline $\boldsymbol{k}$ & $n$ & $\boldsymbol{r}$ & $\boldsymbol{\delta}_{r}$ & $\Upsilon_{r}$ & $\boldsymbol{k}$ & $n$ & $\boldsymbol{r}$ & $\boldsymbol{\delta}_{\boldsymbol{r}}$ & $Y_{r}$ \\
\hline \multirow{42}{*}{2.5} & \multirow[t]{3}{*}{5} & 2 & 0.823503 & -0.51529 & \multirow[t]{23}{*}{2.5} & \multirow[t]{23}{*}{25} & 2 & 0.340376 & -0.14995 \\
\hline & & 3 & 1.062195 & -0.77978 & & & 3 & 0.455794 & -0.23008 \\
\hline & & 4 & 1.272799 & -1.05175 & & & 4 & 0.548491 & -0.30086 \\
\hline & \multirow{8}{*}{10} & 2 & 0.57515 & -0.30804 & & & 5 & 0.627713 & -0.36598 \\
\hline & & 3 & 0.750018 & -0.46366 & & & 6 & 0.697703 & -0.42714 \\
\hline & & 4 & 0.888111 & -0.60149 & & & 7 & 0.760902 & -0.48537 \\
\hline & & 5 & 1.006713 & -0.73168 & & & 8 & 0.81889 & -0.5414 \\
\hline & & 6 & 1.114436 & -0.86044 & & & 9 & 0.872771 & -0.59576 \\
\hline & & 7 & 1.217708 & -0.9943 & & & 10 & 0.923362 & -0.64891 \\
\hline & & 8 & 1.324215 & -1.14403 & & & 11 & 0.971302 & -0.70123 \\
\hline & & 9 & 1.451093 & -1.33836 & & & 12 & 1.017109 & -0.75307 \\
\hline & \multirow[t]{13}{*}{15} & 2 & 0.459334 & -0.22543 & & & 13 & 1.061229 & -0.8048 \\
\hline & & 3 & 0.605895 & -0.34181 & & & 14 & 1.104064 & -0.85677 \\
\hline & & 4 & 0.721962 & -0.44405 & & & 15 & 1.145999 & -0.90941 \\
\hline & & 5 & 0.82062 & -0.53845 & & & 16 & 1.187427 & -0.96319 \\
\hline & & 6 & 0.907894 & -0.62807 & & & 17 & 1.228784 & -1.01873 \\
\hline & & 7 & 0.987327 & -0.71498 & & & 18 & 1.270591 & -1.07684 \\
\hline & & 8 & 1.06135 & -0.80089 & & & 19 & 1.313524 & -1.13868 \\
\hline & & 9 & 1.131893 & -0.8875 & & & 20 & 1.358547 & -1.20596 \\
\hline & & 10 & 1.20077 & -0.97686 & & & 21 & 1.407172 & -1.28153 \\
\hline & & 11 & 1.27007 & -1.07189 & & & 22 & 1.462075 & -1.37057 \\
\hline & & 12 & 1.342836 & -1.17758 & & & 23 & 1.528889 & -1.4842 \\
\hline & & 13 & 1.42488 & -1.3043 & & & 24 & 1.623858 & -1.65491 \\
\hline & & 14 & 1.53241 & -1.48217 & & & & & \\
\hline & \multirow[t]{18}{*}{20} & 2 & 0.388847 & -0.17954 & & & & & \\
\hline & & 3 & 0.517299 & -0.27401 & & & & & \\
\hline & & 4 & 0.619758 & -0.35715 & & & & & \\
\hline & & 5 & 0.707012 & -0.43362 & & & & & \\
\hline & & 6 & 0.784002 & -0.50561 & & & & & \\
\hline & & 7 & 0.853577 & -0.57445 & & & & & \\
\hline & & 8 & 0.917592 & -0.64113 & & & & & \\
\hline & & 9 & 0.977372 & -0.70644 & & & & & \\
\hline & & 10 & 1.033932 & -0.77107 & & & & & \\
\hline & & 11 & 1.088117 & -0.83572 & & & & & \\
\hline & & 12 & 1.140691 & -0.90116 & & & & & \\
\hline & & 13 & 1.192417 & -0.96829 & & & & & \\
\hline & & 14 & 1.244155 & -1.03833 & & & & & \\
\hline & & 15 & 1.297001 & -1.11301 & & & & & \\
\hline & & 16 & 1.352558 & -1.19513 & & & & & \\
\hline & & 17 & 1.413555 & -1.28972 & & & & & \\
\hline & & 18 & 1.485631 & -1.40752 & & & & & \\
\hline & & 19 & 1.584981 & -1.58001 & & & & & \\
\hline
\end{tabular}


Table 3.7: $\quad$ Slope $\left(\boldsymbol{\delta}_{r}\right)$ and Intercept $\left(\Upsilon_{r}\right)$ in Modification to MLE from Censored Sample by Method -I: $k=3.0$

\begin{tabular}{|c|c|c|c|c|c|c|c|c|c|}
\hline $\boldsymbol{k}$ & $n$ & $r$ & $\boldsymbol{\delta}_{r}$ & $\Upsilon_{r}$ & $\boldsymbol{k}$ & $n$ & $r$ & $\boldsymbol{\delta}_{r}$ & $\mathbf{r}_{r}$ \\
\hline \multirow{42}{*}{3.0} & \multirow[t]{3}{*}{5} & 2 & 0.756006 & -0.52925 & \multirow[t]{23}{*}{3.0} & \multirow[t]{23}{*}{25} & 2 & 0.318752 & -0.16504 \\
\hline & & 3 & 0.960339 & -0.77042 & & & 3 & 0.426364 & -0.24819 \\
\hline & & 4 & 1.130073 & -1.00107 & & & 4 & 0.511761 & -0.31999 \\
\hline & \multirow{8}{*}{10} & 2 & 0.534895 & -0.32762 & & & 5 & 0.583937 & -0.3848 \\
\hline & & 3 & 0.69274 & -0.48003 & & & 6 & 0.647017 & -0.44465 \\
\hline & & 4 & 0.813998 & -0.6099 & & & 7 & 0.703372 & -0.50073 \\
\hline & & 5 & 0.915303 & -0.72823 & & & 8 & 0.75453 & -0.55387 \\
\hline & & 6 & 1.00469 & -0.84113 & & & 9 & 0.801555 & -0.60468 \\
\hline & & 7 & 1.087804 & -0.95427 & & & 10 & 0.845229 & -0.65364 \\
\hline & & 8 & 1.170898 & -1.07623 & & & 11 & 0.886153 & -0.70113 \\
\hline & & 9 & 1.267362 & -1.22952 & & & 12 & 0.924814 & -0.74752 \\
\hline & \multirow[t]{13}{*}{15} & 2 & 0.428985 & -0.24373 & & & 13 & 0.961616 & -0.79313 \\
\hline & & 3 & 0.563638 & -0.36103 & & & 14 & 0.996919 & -0.83829 \\
\hline & & 4 & 0.668287 & -0.46109 & & & 15 & 1.031056 & -0.88335 \\
\hline & & 5 & 0.755655 & -0.55109 & & & 16 & 1.064358 & -0.92869 \\
\hline & & 6 & 0.831562 & -0.63447 & & & 17 & 1.09718 & -0.97481 \\
\hline & & 7 & 0.899384 & -0.71341 & & & 18 & 1.129937 & -1.02234 \\
\hline & & 8 & 0.96139 & -0.78958 & & & 19 & 1.163158 & -1.07216 \\
\hline & & 9 & 1.019314 & -0.86453 & & & 20 & 1.197591 & -1.1256 \\
\hline & & 10 & 1.074712 & -0.93995 & & & 21 & 1.234406 & -1.18488 \\
\hline & & 11 & 1.129285 & -1.01818 & & & 22 & 1.275687 & -1.25405 \\
\hline & & 12 & 1.185431 & -1.10308 & & & 23 & 1.325857 & -1.34195 \\
\hline & & 13 & 1.247665 & -1.20273 & & & 24 & 1.397816 & -1.47476 \\
\hline & & 14 & 1.328662 & -1.34098 & & & & & \\
\hline & \multirow[t]{18}{*}{20} & 2 & 0.363827 & -0.19613 & & & & & \\
\hline & & 3 & 0.482922 & -0.293 & & & & & \\
\hline & & 4 & 0.576546 & -0.37613 & & & & & \\
\hline & & 5 & 0.655193 & -0.45096 & & & & & \\
\hline & & 6 & 0.723667 & -0.52002 & & & & & \\
\hline & & 7 & 0.78472 & -0.58484 & & & & & \\
\hline & & 8 & 0.840137 & -0.64649 & & & & & \\
\hline & & 9 & 0.891173 & -0.70579 & & & & & \\
\hline & & 10 & 0.938774 & -0.76342 & & & & & \\
\hline & & 11 & 0.983708 & -0.82003 & & & & & \\
\hline & & 12 & 1.026645 & -0.87627 & & & & & \\
\hline & & 13 & 1.068234 & -0.9329 & & & & & \\
\hline & & 14 & 1.109175 & -0.99087 & & & & & \\
\hline & & 15 & 1.150336 & -1.05153 & & & & & \\
\hline & & 16 & 1.192966 & -1.11703 & & & & & \\
\hline & & 17 & 1.239181 & -1.19129 & & & & & \\
\hline & & 18 & 1.293378 & -1.28277 & & & & & \\
\hline & & 19 & 1.368332 & -1.41665 & & & & & \\
\hline
\end{tabular}


Appendix - V

Table 3.8: $\quad$ Slope $\left(\delta_{r}\right)$ and Intercept $\left(\Upsilon_{r}\right)$ in Modification to MLE from Censored Sample by Method -II: $k=0.25$

\begin{tabular}{|c|c|c|c|c|c|c|c|c|c|}
\hline $\boldsymbol{k}$ & $n$ & $r$ & $\delta_{r}$ & $\mathbf{Y}_{r}$ & $\boldsymbol{k}$ & $n$ & $r$ & $\delta_{r}$ & $\mathbf{Y}_{r}$ \\
\hline \multirow{42}{*}{0.25} & \multirow[t]{3}{*}{5} & 2 & 3.267468 & 0.132721 & \multirow{23}{*}{0.25} & \multirow{23}{*}{25} & 2 & 7.627645 & 0.038197 \\
\hline & & 3 & 3.44044 & 0.094053 & & & 3 & 5.534529 & 0.056735 \\
\hline & & 4 & 4.241724 & -0.20182 & & & 4 & 4.531775 & 0.074491 \\
\hline & \multirow{8}{*}{10} & 2 & 4.095068 & 0.08669 & & & 5 & 3.96987 & 0.091067 \\
\hline & & 3 & 3.414306 & 0.119652 & & & 6 & 3.632716 & 0.105979 \\
\hline & & 4 & 3.245847 & 0.135306 & & & 7 & 3.428117 & 0.118636 \\
\hline & & 5 & 3.329058 & 0.119951 & & & 8 & 3.310425 & 0.128328 \\
\hline & & 6 & 3.595149 & 0.05074 & & & 9 & 3.254772 & 0.134187 \\
\hline & & 7 & 4.04402 & -0.11357 & & & 10 & 3.246787 & 0.135158 \\
\hline & & 8 & 4.73059 & -0.457 & & & 11 & 3.277958 & 0.12994 \\
\hline & & 9 & 5.811663 & -1.18651 & & & 12 & 3.343353 & 0.116917 \\
\hline & \multirow[t]{13}{*}{15} & 2 & 5.220663 & 0.061262 & & & 13 & 3.44044 & 0.094053 \\
\hline & & 3 & 4.025095 & 0.089076 & & & 14 & 3.568497 & 0.058746 \\
\hline & & 4 & 3.517373 & 0.11263 & & & 15 & 3.728354 & 0.007603 \\
\hline & & 5 & 3.300446 & 0.129289 & & & 16 & 3.922384 & -0.06389 \\
\hline & & 6 & 3.244853 & 0.13544 & & & 17 & 4.154731 & -0.16187 \\
\hline & & 7 & 3.298651 & 0.12607 & & & 18 & 4.431818 & -0.295 \\
\hline & & 8 & 3.44044 & 0.094053 & & & 19 & 4.763314 & -0.47585 \\
\hline & & 9 & 3.664566 & 0.028894 & & & 20 & 5.163908 & -0.72359 \\
\hline & & 10 & 3.976672 & -0.08561 & & & 21 & 5.656724 & -1.06909 \\
\hline & & 11 & 4.394443 & -0.27606 & & & 22 & 6.2805 & -1.56615 \\
\hline & & 12 & 4.953784 & -0.58979 & & & 23 & 7.106836 & -2.3194 \\
\hline & & 13 & 5.726488 & -1.12145 & & & 24 & 8.291435 & -3.57152 \\
\hline & & 14 & 6.875098 & -2.09775 & & & & & \\
\hline & \multirow[t]{18}{*}{20} & 2 & 6.411821 & 0.047101 & & & & & \\
\hline & & 3 & 4.758008 & 0.06952 & & & & & \\
\hline & & 4 & 3.990497 & 0.090312 & & & & & \\
\hline & & 5 & 3.585339 & 0.108583 & & & & & \\
\hline & & 6 & 3.368808 & 0.1232 & & & & & \\
\hline & & 7 & 3.267468 & 0.132721 & & & & & \\
\hline & & 8 & 3.245758 & 0.13531 & & & & & \\
\hline & & 9 & 3.285287 & 0.1286 & & & & & \\
\hline & & 10 & 3.376677 & 0.109494 & & & & & \\
\hline & & 11 & 3.516042 & 0.073859 & & & & & \\
\hline & & 12 & 3.703498 & 0.016035 & & & & & \\
\hline & & 13 & 3.94278 & -0.07196 & & & & & \\
\hline & & 14 & 4.241724 & -0.20182 & & & & & \\
\hline & & 15 & 4.613784 & -0.39146 & & & & & \\
\hline & & 16 & 5.081358 & -0.67003 & & & & & \\
\hline & & 17 & 5.683059 & -1.08876 & & & & & \\
\hline & & 18 & 6.491442 & -1.7485 & & & & & \\
\hline & & 19 & 7.666055 & -2.88623 & & & & & \\
\hline
\end{tabular}


Table 3.9: $\quad$ Slope $\left(\boldsymbol{\delta}_{r}\right)$ and Intercept $\left(\Upsilon_{r}\right)$ in Modification to MLE from Censored Sample by Method -II: $k=0.50$

\begin{tabular}{|c|c|c|c|c|c|c|c|c|c|}
\hline$k$ & $n$ & $r$ & $\delta_{r}$ & $\mathbf{Y}_{r}$ & $\boldsymbol{k}$ & $n$ & $r$ & $\delta_{r}$ & $\mathbf{Y}_{r}$ \\
\hline \multirow{42}{*}{0.50} & \multirow[t]{3}{*}{5} & 2 & 1.775421 & -0.13818 & \multirow{23}{*}{0.50} & \multirow{23}{*}{25} & 2 & 1.158527 & -0.00616 \\
\hline & & 3 & 2.292353 & -0.36648 & & & 3 & 1.241604 & -0.01418 \\
\hline & & 4 & 2.988413 & -0.82167 & & & 4 & 1.327531 & -0.02581 \\
\hline & \multirow{8}{*}{10} & 2 & 1.391967 & -0.03671 & & & 5 & 1.41658 & -0.04135 \\
\hline & & 3 & 1.614299 & -0.0881 & & & 6 & 1.509064 & -0.06115 \\
\hline & & 4 & 1.860575 & -0.16884 & & & 7 & 1.605344 & -0.08563 \\
\hline & & 5 & 2.138313 & -0.28809 & & & 8 & 1.705841 & -0.11527 \\
\hline & & 6 & 2.459047 & -0.46074 & & & 9 & 1.811046 & -0.15066 \\
\hline & & 7 & 2.841793 & -0.71263 & & & 10 & 1.921537 & -0.19251 \\
\hline & & 8 & 3.321357 & -1.09387 & & & 11 & 2.038004 & -0.24168 \\
\hline & & 9 & 3.973092 & -1.72028 & & & 12 & 2.161274 & -0.29924 \\
\hline & \multirow[t]{13}{*}{15} & 2 & 1.262809 & -0.01674 & & & 13 & 2.292353 & -0.36648 \\
\hline & & 3 & 1.405268 & -0.03918 & & & 14 & 2.432481 & -0.44507 \\
\hline & & 4 & 1.556705 & -0.07278 & & & 15 & 2.583211 & -0.53714 \\
\hline & & 5 & 1.718723 & -0.11937 & & & 16 & 2.746521 & -0.64544 \\
\hline & & 6 & 1.893383 & -0.1814 & & & 17 & 2.92499 & -0.77367 \\
\hline & & 7 & 2.083383 & -0.26222 & & & 18 & 3.122069 & -0.9269 \\
\hline & & 8 & 2.292353 & -0.36648 & & & 19 & 3.342526 & -1.1123 \\
\hline & & 9 & 2.52533 & -0.50088 & & & 20 & 3.593223 & -1.34053 \\
\hline & & 10 & 2.7896 & -0.67546 & & & 21 & 3.884577 & -1.62822 \\
\hline & & 11 & 3.09627 & -0.90616 & & & 22 & 4.233591 & -2.00349 \\
\hline & & 12 & 3.463551 & -1.22021 & & & 23 & 4.671077 & -2.51926 \\
\hline & & 13 & 3.924596 & -1.66958 & & & 24 & 5.263125 & -3.29443 \\
\hline & & 14 & 4.550783 & -2.37251 & & & & & \\
\hline & \multirow{18}{*}{20} & 2 & 1.197748 & -0.00955 & & & & & \\
\hline & & 3 & 1.302675 & -0.0221 & & & & & \\
\hline & & 4 & 1.412264 & -0.04052 & & & & & \\
\hline & & 5 & 1.527098 & -0.06544 & & & & & \\
\hline & & 6 & 1.647871 & -0.09767 & & & & & \\
\hline & & 7 & 1.775421 & -0.13818 & & & & & \\
\hline & & 8 & 1.910768 & -0.18823 & & & & & \\
\hline & & 9 & 2.055176 & -0.24937 & & & & & \\
\hline & & 10 & 2.210227 & -0.32363 & & & & & \\
\hline & & 11 & 2.377941 & -0.41367 & & & & & \\
\hline & & 12 & 2.56096 & -0.52307 & & & & & \\
\hline & & 13 & 2.762818 & -0.65673 & & & & & \\
\hline & & 14 & 2.988413 & -0.82167 & & & & & \\
\hline & & 15 & 3.244802 & -1.02832 & & & & & \\
\hline & & 16 & 3.542721 & -1.2931 & & & & & \\
\hline & & 17 & 3.899716 & -1.64382 & & & & & \\
\hline & & 18 & 4.347643 & -2.13316 & & & & & \\
\hline & & 19 & 4.954907 & -2.88001 & & & & & \\
\hline
\end{tabular}


Table 3.10: Slope $\left(\delta_{r}\right)$ and Intercept $\left(\Upsilon_{r}\right)$ in Modification to MLE from Censored Sample by Method -II: $k=1.50$

\begin{tabular}{|c|c|c|c|c|c|c|c|c|c|}
\hline $\boldsymbol{k}$ & $n$ & $r$ & $\boldsymbol{\delta}_{r}$ & $\mathbf{Y}_{r}$ & $\boldsymbol{k}$ & $n$ & $r$ & $\delta_{r}$ & $\mathbf{Y}_{r}$ \\
\hline \multirow{42}{*}{1.50} & 5 & 2 & 1.075006 & -0.51634 & \multirow{23}{*}{1.50} & \multirow{23}{*}{25} & 2 & 0.460274 & -0.13029 \\
\hline & & 3 & 1.374915 & -0.78692 & & & 3 & 0.583236 & -0.18976 \\
\hline & & 4 & 1.675123 & -1.1163 & & & 4 & 0.688548 & -0.24778 \\
\hline & \multirow{8}{*}{10} & 2 & 0.757851 & -0.2895 & & & 5 & 0.782621 & -0.30509 \\
\hline & & 3 & 0.956607 & -0.4247 & & & 6 & 0.868898 & -0.36218 \\
\hline & & 4 & 1.131581 & -0.56311 & & & 7 & 0.949507 & -0.41947 \\
\hline & & 5 & 1.294999 & -0.7093 & & & 8 & 1.025894 & -0.47731 \\
\hline & & 6 & 1.454811 & -0.86867 & & & 9 & 1.099116 & -0.53604 \\
\hline & & 7 & 1.618304 & -1.04925 & & & 10 & 1.169994 & -0.59599 \\
\hline & & 8 & 1.795003 & -1.2653 & & & 11 & 1.239203 & -0.65751 \\
\hline & & 9 & 2.002707 & -1.54842 & & & 12 & 1.30733 & -0.72101 \\
\hline & \multirow[t]{13}{*}{15} & 2 & 0.610889 & -0.20436 & & & 13 & 1.374915 & -0.78692 \\
\hline & & 3 & 0.771343 & -0.29794 & & & 14 & 1.442485 & -0.85578 \\
\hline & & 4 & 0.909809 & -0.39078 & & & 15 & 1.510586 & -0.92824 \\
\hline & & 5 & 1.035201 & -0.4846 & & & 16 & 1.579818 & -1.0051 \\
\hline & & 6 & 1.152455 & -0.58086 & & & 17 & 1.650884 & -1.08742 \\
\hline & & 7 & 1.264848 & -0.68107 & & & 18 & 1.72465 & -1.17661 \\
\hline & & 8 & 1.374915 & -0.78692 & & & 19 & 1.802254 & -1.27464 \\
\hline & & 9 & 1.484949 & -0.9006 & & & 20 & 1.885286 & -1.3844 \\
\hline & & 10 & 1.597383 & -1.02512 & & & 21 & 1.976123 & -1.51037 \\
\hline & & 11 & 1.715246 & -1.16503 & & & 22 & 2.078649 & -1.66011 \\
\hline & & 12 & 1.842967 & -1.32783 & & & 23 & 2.199996 & -1.84789 \\
\hline & & 13 & 1.988195 & -1.52758 & & & 24 & 2.355862 & -2.10621 \\
\hline & & 14 & 2.167279 & -1.79612 & & & & & \\
\hline & \multirow{18}{*}{20} & 2 & 0.521653 & -0.15885 & & & & & \\
\hline & & 3 & 0.659807 & -0.2313 & & & & & \\
\hline & & 4 & 0.778339 & -0.30237 & & & & & \\
\hline & & 5 & 0.884638 & -0.37307 & & & & & \\
\hline & & 6 & 0.982692 & -0.44417 & & & & & \\
\hline & & 7 & 1.075006 & -0.51634 & & & & & \\
\hline & & 8 & 1.163325 & -0.59021 & & & & & \\
\hline & & 9 & 1.248989 & -0.66645 & & & & & \\
\hline & & 10 & 1.333112 & -0.74581 & & & & & \\
\hline & & 11 & 1.416712 & -0.82917 & & & & & \\
\hline & & 12 & 1.500803 & -0.91764 & & & & & \\
\hline & & 13 & 1.586495 & -1.01269 & & & & & \\
\hline & & 14 & 1.675123 & -1.1163 & & & & & \\
\hline & & 15 & 1.768437 & -1.23139 & & & & & \\
\hline & & 16 & 1.868956 & -1.36241 & & & & & \\
\hline & & 17 & 1.9807 & -1.51689 & & & & & \\
\hline & & 18 & 2.110952 & -1.70897 & & & & & \\
\hline & & 19 & 2.27564 & -1.97083 & & & & & \\
\hline
\end{tabular}


Table 3.11: Slope $\left(\delta_{r}\right)$ and Intercept $\left(\Upsilon_{r}\right)$ in Modification to MLE from Censored Sample by Method -II: $k=2.0$

\begin{tabular}{|c|c|c|c|c|c|c|c|c|c|}
\hline$k$ & $n$ & $r$ & $\boldsymbol{\delta}_{r}$ & $\mathbf{Y}_{r}$ & & $n$ & $\boldsymbol{r}$ & $\delta_{r}$ & $\mathbf{Y}_{r}$ \\
\hline \multirow{42}{*}{2.0} & \multirow[t]{3}{*}{5} & 2 & 0.950885 & -0.56721 & \multirow{23}{*}{2.0} & \multirow{23}{*}{25} & 2 & 0.411493 & -0.164 \\
\hline & & 3 & 1.191522 & -0.81173 & & & 3 & 0.523443 & -0.23198 \\
\hline & & 4 & 1.416142 & -1.08187 & & & 4 & 0.617976 & -0.29579 \\
\hline & \multirow{8}{*}{10} & 2 & 0.679382 & -0.34035 & & & 5 & 0.701163 & -0.35674 \\
\hline & & 3 & 0.851468 & -0.47838 & & & 6 & 0.776299 & -0.41568 \\
\hline & & 4 & 0.997524 & -0.61126 & & & 7 & 0.84543 & -0.4732 \\
\hline & & 5 & 1.129003 & -0.74399 & & & 8 & 0.90994 & -0.5298 \\
\hline & & 6 & 1.252859 & -0.88122 & & & 9 & 0.97083 & -0.58586 \\
\hline & & 7 & 1.374824 & -1.02886 & & & 10 & 1.028866 & -0.64174 \\
\hline & & 8 & 1.501658 & -1.1967 & & & 11 & 1.084659 & -0.69778 \\
\hline & & 9 & 1.645321 & -1.40606 & & & 12 & 1.138726 & -0.75433 \\
\hline & \multirow[t]{13}{*}{15} & 2 & 0.548399 & -0.24827 & & & 13 & 1.191522 & -0.81173 \\
\hline & & 3 & 0.691257 & -0.34925 & & & 14 & 1.243472 & -0.87039 \\
\hline & & 4 & 0.811518 & -0.44458 & & & 15 & 1.294995 & -0.93077 \\
\hline & & 5 & 0.917732 & -0.53683 & & & 16 & 1.346532 & -0.99345 \\
\hline & & 6 & 1.014589 & -0.62777 & & & 17 & 1.398581 & -1.05915 \\
\hline & & 7 & 1.105111 & -0.71891 & & & 18 & 1.451735 & -1.12881 \\
\hline & & 8 & 1.191522 & -0.81173 & & & 19 & 1.506763 & -1.20378 \\
\hline & & 9 & 1.275696 & -0.90789 & & & 20 & 1.564722 & -1.28598 \\
\hline & & 10 & 1.359475 & -1.00956 & & & 21 & 1.627195 & -1.37846 \\
\hline & & 11 & 1.445006 & -1.11985 & & & 22 & 1.696778 & -1.48637 \\
\hline & & 12 & 1.535293 & -1.24382 & & & 23 & 1.778282 & -1.61956 \\
\hline & & 13 & 1.635435 & -1.39096 & & & 24 & 1.882446 & -1.80076 \\
\hline & & 14 & 1.75637 & -1.58302 & & & & & \\
\hline & \multirow{18}{*}{20} & 2 & 0.467561 & -0.19701 & & & & & \\
\hline & & 3 & 0.592316 & -0.2779 & & & & & \\
\hline & & 4 & 0.697404 & -0.35389 & & & & & \\
\hline & & 5 & 0.789881 & -0.42673 & & & & & \\
\hline & & 6 & 0.873578 & -0.49755 & & & & & \\
\hline & & 7 & 0.950885 & -0.56721 & & & & & \\
\hline & & 8 & 1.023444 & -0.63642 & & & & & \\
\hline & & 9 & 1.092478 & -0.70582 & & & & & \\
\hline & & 10 & 1.158965 & -0.77607 & & & & & \\
\hline & & 11 & 1.223755 & -0.84786 & & & & & \\
\hline & & 12 & 1.287644 & -0.92202 & & & & & \\
\hline & & 13 & 1.351459 & -0.99957 & & & & & \\
\hline & & 14 & 1.416142 & -1.08187 & & & & & \\
\hline & & 15 & 1.482891 & -1.17089 & & & & & \\
\hline & & 16 & 1.553392 & -1.26964 & & & & & \\
\hline & & 17 & 1.630321 & -1.3832 & & & & & \\
\hline & & 18 & 1.718545 & -1.52121 & & & & & \\
\hline & & 19 & 1.828849 & -1.70597 & & & & & \\
\hline
\end{tabular}


Table 3.12: Slope $\left(\delta_{r}\right)$ and Intercept $\left(\Upsilon_{r}\right)$ in Modification to MLE from Censored Sample by Method -II: $k=2.5$

\begin{tabular}{|c|c|c|c|c|c|c|c|c|c|}
\hline $\boldsymbol{k}$ & $n$ & $r$ & $\delta_{r}$ & $\mathbf{Y}_{r}$ & $\boldsymbol{k}$ & $n$ & $r$ & $\delta_{r}$ & $\mathbf{Y}_{r}$ \\
\hline \multirow{42}{*}{2.5} & 5 & 2 & 0.861412 & -0.59062 & \multirow{23}{*}{2.5} & \multirow{23}{*}{25} & 2 & 0.380247 & -0.18713 \\
\hline & & 3 & 1.061074 & -0.81049 & & & 3 & 0.483127 & -0.25948 \\
\hline & & 4 & 1.236938 & -1.03581 & & & 4 & 0.568948 & -0.32557 \\
\hline & \multirow{8}{*}{10} & 2 & 0.624105 & -0.37079 & & & 5 & 0.643548 & -0.38724 \\
\hline & & 3 & 0.775859 & -0.5065 & & & 6 & 0.710111 & -0.44563 \\
\hline & & 4 & 0.90096 & -0.63146 & & & 7 & 0.77061 & -0.50152 \\
\hline & & 5 & 1.010278 & -0.75121 & & & 8 & 0.826378 & -0.55548 \\
\hline & & 6 & 1.110135 & -0.87009 & & & 9 & 0.878372 & -0.60798 \\
\hline & & 7 & 1.205369 & -0.99288 & & & 10 & 0.927315 & -0.6594 \\
\hline & & 8 & 1.301218 & -1.1269 & & & 11 & 0.973778 & -0.71008 \\
\hline & & 9 & 1.406477 & -1.28769 & & & 12 & 1.018229 & -0.76034 \\
\hline & \multirow[t]{13}{*}{15} & 2 & 0.505884 & -0.2765 & & & 13 & 1.061074 & -0.81049 \\
\hline & & 3 & 0.634713 & -0.37973 & & & 14 & 1.102677 & -0.86088 \\
\hline & & 4 & 0.741024 & -0.47385 & & & 15 & 1.143386 & -0.91187 \\
\hline & & 5 & 0.833067 & -0.56212 & & & 16 & 1.183553 & -0.96389 \\
\hline & & 6 & 0.915332 & -0.64663 & & & 17 & 1.223562 & -1.01748 \\
\hline & & 7 & 0.99066 & -0.72896 & & & 18 & 1.26386 & -1.07334 \\
\hline & & 8 & 1.061074 & -0.81049 & & & 19 & 1.305013 & -1.13244 \\
\hline & & 9 & 1.128203 & -0.89265 & & & 20 & 1.347792 & -1.19618 \\
\hline & & 10 & 1.193554 & -0.97711 & & & 21 & 1.393349 & -1.2668 \\
\hline & & 11 & 1.25879 & -1.0662 & & & 22 & 1.443579 & -1.34808 \\
\hline & & 12 & 1.326139 & -1.16362 & & & 23 & 1.502026 & -1.44738 \\
\hline & & 13 & 1.399321 & -1.27627 & & & 24 & 1.576698 & -1.58188 \\
\hline & & 14 & 1.486334 & -1.42021 & & & & & \\
\hline & \multirow{18}{*}{20} & 2 & 0.431927 & -0.22253 & & & & & \\
\hline & & 3 & 0.545758 & -0.3072 & & & & & \\
\hline & & 4 & 0.640197 & -0.38439 & & & & & \\
\hline & & 5 & 0.722055 & -0.45645 & & & & & \\
\hline & & 6 & 0.795028 & -0.52485 & & & & & \\
\hline & & 7 & 0.861412 & -0.59062 & & & & & \\
\hline & & 8 & 0.922769 & -0.65454 & & & & & \\
\hline & & 9 & 0.980241 & -0.71727 & & & & & \\
\hline & & 10 & 1.03472 & -0.77944 & & & & & \\
\hline & & 11 & 1.086952 & -0.84163 & & & & & \\
\hline & & 12 & 1.137612 & -0.90453 & & & & & \\
\hline & & 13 & 1.187364 & -0.96891 & & & & & \\
\hline & & 14 & 1.236938 & -1.03581 & & & & & \\
\hline & & 15 & 1.287228 & -1.10664 & & & & & \\
\hline & & 16 & 1.339472 & -1.1836 & & & & & \\
\hline & & 17 & 1.395615 & -1.27039 & & & & & \\
\hline & & 18 & 1.459215 & -1.37414 & & & & & \\
\hline & & 19 & 1.53824 & -1.51153 & & & & & \\
\hline
\end{tabular}


Table 3.13: Slope $\left(\delta_{r}\right)$ and Intercept $\left(\Upsilon_{r}\right)$ in Modification to MLE from Censored Sample by Method -II: $k=3.0$

\begin{tabular}{|c|c|c|c|c|c|c|c|c|c|}
\hline $\boldsymbol{k}$ & $n$ & $r$ & $\delta_{r}$ & $Y_{r}$ & $\boldsymbol{k}$ & $n$ & $\boldsymbol{r}$ & $\delta_{r}$ & $\mathbf{Y}_{r}$ \\
\hline \multirow{42}{*}{3.0} & \multirow[t]{3}{*}{5} & 2 & 0.792232 & -0.59996 & \multirow{23}{*}{3.0} & \multirow{23}{*}{25} & 2 & 0.357065 & -0.20337 \\
\hline & & 3 & 0.961963 & -0.7983 & & & 3 & 0.452459 & -0.27784 \\
\hline & & 4 & 1.103985 & -0.98907 & & & 4 & 0.531195 & -0.34448 \\
\hline & \multirow{8}{*}{10} & 2 & 0.581344 & -0.38937 & & & 5 & 0.59893 & -0.40557 \\
\hline & & 3 & 0.717204 & -0.52084 & & & 6 & 0.658748 & -0.46247 \\
\hline & & 4 & 0.826482 & -0.63772 & & & 7 & 0.712562 & -0.5161 \\
\hline & & 5 & 0.91957 & -0.74603 & & & 8 & 0.761658 & -0.56713 \\
\hline & & 6 & 1.002343 & -0.84997 & & & 9 & 0.806955 & -0.61606 \\
\hline & & 7 & 1.079056 & -0.95366 & & & 10 & 0.849143 & -0.66331 \\
\hline & & 8 & 1.153999 & -1.06288 & & & 11 & 0.888761 & -0.70923 \\
\hline & & 9 & 1.234031 & -1.18959 & & & 12 & 0.926244 & -0.75413 \\
\hline & \multirow[t]{13}{*}{15} & 2 & 0.473417 & -0.29512 & & & 13 & 0.961963 & -0.7983 \\
\hline & & 3 & 0.590945 & -0.39819 & & & 14 & 0.996242 & -0.84204 \\
\hline & & 4 & 0.686315 & -0.48965 & & & 15 & 1.029382 & -0.88566 \\
\hline & & 5 & 0.767512 & -0.57335 & & & 16 & 1.061682 & -0.9295 \\
\hline & & 6 & 0.838856 & -0.65164 & & & 17 & 1.093453 & -0.974 \\
\hline & & 7 & 0.903046 & -0.72617 & & & 18 & 1.125052 & -1.01969 \\
\hline & & 8 & 0.961963 & -0.7983 & & & 19 & 1.156921 & -1.06732 \\
\hline & & 9 & 1.017069 & -0.86929 & & & 20 & 1.189658 & -1.11796 \\
\hline & & 10 & 1.069661 & -0.94055 & & & 21 & 1.224149 & -1.17333 \\
\hline & & 11 & 1.121098 & -1.01389 & & & 22 & 1.261861 & -1.23637 \\
\hline & & 12 & 1.173135 & -1.09218 & & & 23 & 1.035553 & -1.31285 \\
\hline & & 13 & 1.228647 & -1.18071 & & & 24 & 1.361532 & -1.41644 \\
\hline & & 14 & 1.293826 & -1.29196 & & & & & \\
\hline & \multirow{18}{*}{20} & 2 & 0.405112 & -0.24001 & & & & & \\
\hline & & 3 & 0.510002 & -0.32609 & & & & & \\
\hline & & 4 & 0.595903 & -0.40277 & & & & & \\
\hline & & 5 & 0.669416 & -0.47292 & & & & & \\
\hline & & 6 & 0.734121 & -0.53826 & & & & & \\
\hline & & 7 & 0.792232 & -0.59996 & & & & & \\
\hline & & 8 & 0.845244 & -0.65887 & & & & & \\
\hline & & 9 & 0.894237 & -0.7157 & & & & & \\
\hline & & 10 & 0.940041 & -0.77103 & & & & & \\
\hline & & 11 & 0.983333 & -0.82541 & & & & & \\
\hline & & 12 & 1.024706 & -0.87942 & & & & & \\
\hline & & 13 & 1.064725 & -0.9337 & & & & & \\
\hline & & 14 & 1.103985 & -0.98907 & & & & & \\
\hline & & 15 & 1.143196 & -1.04661 & & & & & \\
\hline & & 16 & 1.18332 & -1.10802 & & & & & \\
\hline & & 17 & 1.225857 & -1.17613 & & & & & \\
\hline & & 18 & 1.273558 & -1.25648 & & & & & \\
\hline & & 19 & 1.332648 & -1.3622 & & & & & \\
\hline
\end{tabular}




\section{Appendix- VI}

Table 3.14: Empirical Variances of MMLE of $\lambda$ from Censored Sample: Method I \& Method -II

\begin{tabular}{|c|c|c|c|c|c|c|}
\hline \multicolumn{7}{|c|}{ MMLE: Method -I } \\
\hline & 0.25 & 0.50 & 1.50 & 2.0 & 2.5 & 3.0 \\
\hline$(5,4)$ & 11.49365 & 0.412213 & 0.18131 & 0.187485 & 0.21507 & 0.245198 \\
\hline$(\mathbf{1 0 , 8 )}$ & 0.410329 & 0.11826 & 0.103083 & 0.125636 & 0.150761 & 0.182708 \\
\hline$(15,12)$ & 0.138162 & 0.075133 & 0.040271 & 0.109317 & 0.131422 & 0.16653 \\
\hline$(20,16)$ & 0.092643 & 0.055522 & 0.071039 & 0.091347 & 0.12222 & 0.150345 \\
\hline$(25,20)$ & 0.067846 & 0.042435 & 0.067635 & 0.089057 & 0.114548 & 0.150973 \\
\hline \multicolumn{7}{|c|}{ MMLE: Method -II } \\
\hline & 0.25 & 0.50 & 1.50 & 2.0 & 2.5 & 3.0 \\
\hline$(5,4)$ & 5.027481 & 0.388384 & 0.179044 & 0.193406 & 0.21721 & 0.737996 \\
\hline$(\mathbf{1 0 , 8})$ & 0.534766 & 0.103742 & 0.100408 & 0.120603 & 0.151993 & 0.180261 \\
\hline$(15,12)$ & 0.180459 & 0.065647 & 0.082163 & 0.102875 & 0.130453 & 0.162365 \\
\hline$(20,16)$ & 0.109447 & 0.047965 & 0.072703 & 0.092277 & 0.122778 & 0.149991 \\
\hline$(25,20)$ & 0.083155 & 0.038627 & 0.066796 & 0.084847 & 0.113837 & 0.146095 \\
\hline
\end{tabular}

\section{References}

1. Bhattacharyya, G.K. (1985). The Asymptotic of Maximum Likelihood and Related Estimators based on Type II Censored Data. Journal of American Statistical Association, Vol.80, 398-404.

2. Burr, I.W. (1942). Cumulative Frequency Functions. The Annals of Mathematical Statistics, Vol.13, 215-232.

3. Gupta, R.D., and Kundu D. (1999). Generalized Exponential Distributions. Australian and New Zealand Journal of Statistics, Vol.41, No.2, 173-188.

4. Kantam, R.R.L., Priya, M.Ch. and Ravikumar, M.S. (2013). Modified Maximum Likelihood Estimation in Linear Failure Rate Distribution. Inter Stat, July.

5. Katnam, R.R.L. and Ravikumar, M.S. (2015). Modified Maximum Likelihood Estimation in Burr Type X Distribution. Inter Stat, June, 1-14.

6. Kantam, R.R.L., and Srinivasa Rao, G. (1993). Reliability Estimation in Rayleigh Distribution with Censoring - Some Approximations to ML Method. Proceedings of II Annual Conference of Society for Development of Statistics, 58-63. 
7. Kantam, R.R.L., and Srinivasa Rao, G. (2002). Log-logistic Distribution: Modified Maximum Likelihood Estimation. Gujarat Statistical Review, Vol. 29, No. $1 \&$ 2, 25-36.

8. Kantam, R.R.L., and Sriram, B. (2003). Maximum Likelihood Estimation from Censored Samples - Some Modifications in Length Biased Version of Exponential Model. Statistical Methods, Vol. 5, No. 1, 63-78.

9. Kundu, D., and Raqab, M. Z. (2005). Generalized Rayleigh Distribution: Different Methods of Estimation. Computational Statistics \& Data Analysis, Vol. 49, 187-200.

10. Mehrotra, K.G., and Nanda, P. (1974): Unbiased Estimation of Parameters by Order Statistics in the case of Censored Samples, Biometrika, Vol. 61, 601-606.

11. Mudholkar, Govind S., and Srivastava, Deo Kumar. (1993). Exponentiated Weibull family for analyzing bathtub failure-rate. Reliability, IEEE Transaction, Vol.42, No.2, 299 - 302.

12. Pearson, E.S., and Rootzen, H. (1977). Simple and Highly Efficient Estimators for a Type-I Censored Normal Sample. Biometrika, Vol. 64, No. 1, 123-128.

13. Rao, C.R., Mathai, A., and Mitra, S.K. (1966). Formulae and Tables for Statistical Work. Statistical Publishing Society, Calcutta, India.

14. Raqab, M. Z., and Kundu, D. (2006). Burr type X distribution: revisited. Journal of Probability and Statistical Science, Vol.8, 179-198.

15. Rosaiah, K., Kantam, R.R.L., and Narasimham, V.L. (1993a). ML and Modified ML Estimation in Gamma Distribution with a Known Prior Relation Among the Parameters. Pakistan Journal of Statistics, Vol. 9, No. 3(B), 37-48.

16. Rosaiah, K., Kantam, R.R.L., and Narasimham, V.L. (1993b). On Modified Maximum Likelihood Estimation of Gamma Parameters. Journal of Statistical Research, Bangladesh, Vol. 27, No. 1\&2, 15-28.

17. Tiku, M.L (1967). Estimating the Mean and Standard Deviation from a Censored Normal Sample. Biometrika, Vol. 54, 155-165.

18. Tiku, M.L., and Suresh, R.P. (1992). A New Method of Estimation for Location and Scale Parameters. Journal of Statistical Planning \& Inference, Vol. 30, 281-292.

19. Tiku, M.L., Tan, W.Y., and Balakrishnan, N. (1986). Robust Inference. Marcel Dekker. New York. 